\title{
Polarimetric Channel Characterization of Foliage for Performance Assessment of GPS Receivers Under Tree Canopies
}

\author{
Il-Suek Koh, Associate Member, IEEE, and Kamal Sarabandi, Fellow, IEEE
}

\begin{abstract}
The attenuation, depolarization, and fluctuation of a microwave signal going through a tree canopy are investigated by developing a Monte Carlo based coherent scattering model. In particular, the model is used to analyze the performance of global positioning system (GPS) receivers under tree canopies. Also the frequency and time-domain channel characteristics of a forest are investigated when a transmitter is outside and a receiver is inside a forest. A fractal algorithm (Lindenmayer system) is used to generate the structure of coniferous or deciduous trees whose basic building blocks are arbitrarily oriented finite cylinders, thin dielectric needles, and thin dielectric disks. Attenuation and phase change of the mean field through foliage is accounted for using Foldy's approximation. Scattering of the mean field from individual tree components and their images in the underlying ground plane are computed analytically and added coherently. Since tree trunks and some branches are large compared to the wavelength and may be in the close proximity of the receiver, a closed-form and uniform expression for the scattered near-field from dielectric cylinders is also developed. Monte Carlo simulation of field calculation is applied to a cluster of trees in order to estimate the statistics of the channel parameters, such as the probability density function (pdf) of the polarization state of the transmitted field, path loss, and the incoherent scattered power (the second moment of the scattered field), as a function of the observation point above the ground.
\end{abstract}

Index Terms-Channel simulation, propagation, vegetation.

\section{INTRODUCTION}

W ITH the increasing demand for wireless communications, the need for the efficient use of the spectrum is felt more than ever. Accurate estimation of system requirements such as transmitter power, bandwidth, modulation scheme, etc., all depend on the communication channel characteristics. Ground-to-air/satellite communication is a scenario with numerous civilian and military applications ranging from simple voice and data communications to navigation. In this scenario, characterization of the channel is rather straightforward except for the urban environment or forested areas where significant scattering and shadowing may take place. In this paper, the problem of channel characterization for a forested environment is considered. Using this model, performance of

Manuscript received June 15, 2001; revised November 15, 2001. This work was supported in part by the National Science Foundation, under Contact ECS-9 979376 and in part by the U.S. Army Research Office under Contract DAAH04-96-1-0377.

The authors are with the Radiation Laboratory, Department of Electrical Engineering and Computer Science, The University of Michigan, Ann Arbor, MI 48109-2122 USA (e-mail: saraband@eecs.umich.edu).

Publisher Item Identifier S 0018-926X(02)05458-3. global positioning system (GPS) receivers under tree canopies and a narrow pulse transmission through a tree canopy are investigated as examples.

The GPS was introduced by the Department of Defense (DoD) primarily for the U.S. military to provide precise estimation of position, velocity, and time. Since the early 1980s, the DoD has allowed GPS to be used for civilian applications. Because of the high price of GPS receivers in early years, the application of GPS for commercial purposes was limited mainly to civil aviation. In recent years, however, mainly with the rapid development of the integrated circuit, the availability of low-cost receivers has significantly broadened the application domain of the GPS, such as land transportation, surveying and mapping, agriculture, earth sciences, etc. [1]. Each GPS satellite transmits a right hand circularly polarized $L$-band signal at two frequency bands known as $L 1(1.57542 \mathrm{GHz})$ and $L 2(1.2276 \mathrm{GHz})$. For a commercial use, the $L 1$ signal is modulated by a pseudo random noise (PRN) code called the coarse/ccquisition $(\mathrm{C} / \mathrm{P})$ code which is a 1023 gold code with a chip rate of 1023 microchips/s (Mcps) and its null-to-null bandwidth is $2.046 \mathrm{MHz}$ [2]. When this signal reaches the earth's surface, the level is very weak and therefore the receiver performance may be severely degraded if a line-of-sight (LOS) transmission is not established.

With the advent of powerful computers, physics-based approaches for predicting deterministic or statistical channel characteristics have attained significant prominence in recent years [3]. These models are more comprehensive and can be used for a larger class of scenarios with a high accuracy than traditional models based on measurements or simple diffraction models. However, the physics-based models are complex and not easy to use because of the difficulty of electromagnetic modeling of an environment. In this study, the development of a physics-based channel model for vegetation canopies is considered. The literature concerning interaction of electromagnetic waves and vegetation can be categorized into two groups, namely, papers related to radar remote sensing of vegetation [4]-[8] and papers related to communications [9]-[11]. The thrust of remote sensing articles is on the modeling of vegetation scattering when both the transmitter and receiver are in the far field region of the illuminated canopy and the interest is usually in the calculation of the mean backscatter power. In the communication problem where the transmitter or receiver, or both, are within the canopy, the field calculations and the mechanisms responsible for the dominant field at the receiver are very different. For example, when both the transmitter and receiver are embedded in the 


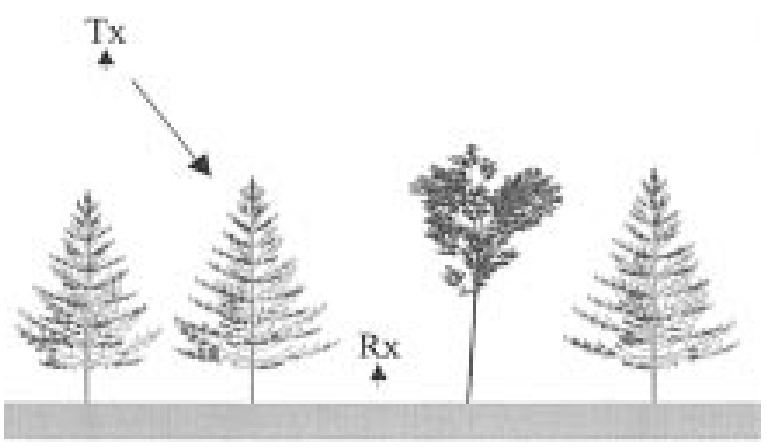

Fig. 1. The problem geometry where a receiver inside a forest is communicating with a transmitter above.

canopy, there is a nonvanishing dominant mean-field at frequencies below UHF, which can be attributed to a lateral wave which propagates along the canopy-air interface and sheds energy at critical angle. Mathematically this lateral wave results from the branch-cut contribution of the spectral representation of a dipole field in a dielectric slab [9], [10]. Wave propagation over relatively long distances at UHF and higher where both the transmitter and receiver are embedded in a forest canopy is not possible due to exorbitant propagation path loss. At these frequencies either the transmitter or the receiver must be outside the canopy. Field calculations for this scenario are complicated because of the close proximity of the receiver (transmitter) to relatively large scatterers, which can cause significant field attenuation and fluctuation.

In what follows, development of an accurate model that describes the propagation of electromagnetic waves through foliage is considered. In Section II, the overall structure of the coherent channel model based on a single scattering theory and Monte Carlo simulation is presented. Also, an analytical approximate scattering formulation for an arbitrarily oriented finite dielectric cylinder above a ground plane, which can represent tree trunks and branches, is provided. This new formulation is a building block for the channel model and provides a uniform expression for the scattered field which is valid for observation points near and far from the cylinder. Finally in Section III, numerical simulations are presented showing the effects of foliage on the GPS signal. Quantities such as the signal path loss, statistics of the received polarization, and the first-order statistics of the electric field for both horizontally and vertically polarized incident waves are calculated. In addition, the effect of the tree canopy on an ultra-wide-band signal is investigated.

\section{FOREST MODEL}

From electromagnetic scattering point of view, a tree can be considered as a complex structure composed of a group of randomly oriented scatterers structured in a semideterministic fashion with electrical characteristics very much dependent on their moisture content. At UHF frequencies and higher, dimensions of tree trunk, the primary and secondary branches, or even leaves and twigs can be much larger than or comparable to the wavelength. Noting that a relatively large cluster of trees around an observation point significantly contribute to the total field at the receiver, field calculations based on brute-force numerical techniques are not possible at microwave frequencies or higher.
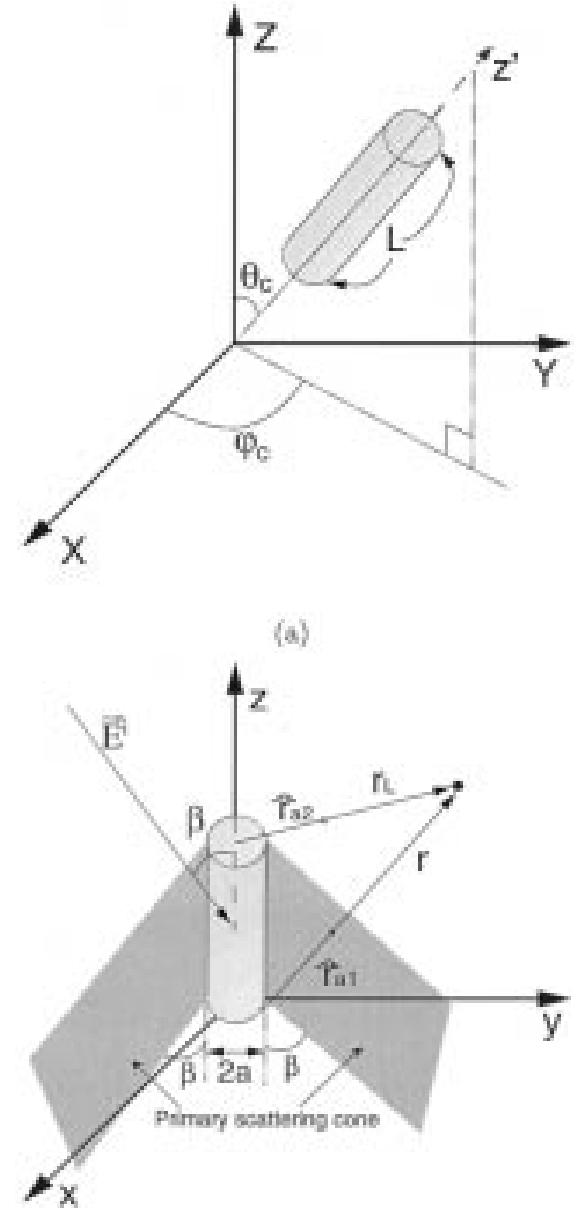

(b)

Fig. 2. Global and local coordinate systems for an arbitrarily oriented cylinder. (a) Before the coordinate transformation. (b) After the coordinate transformation.
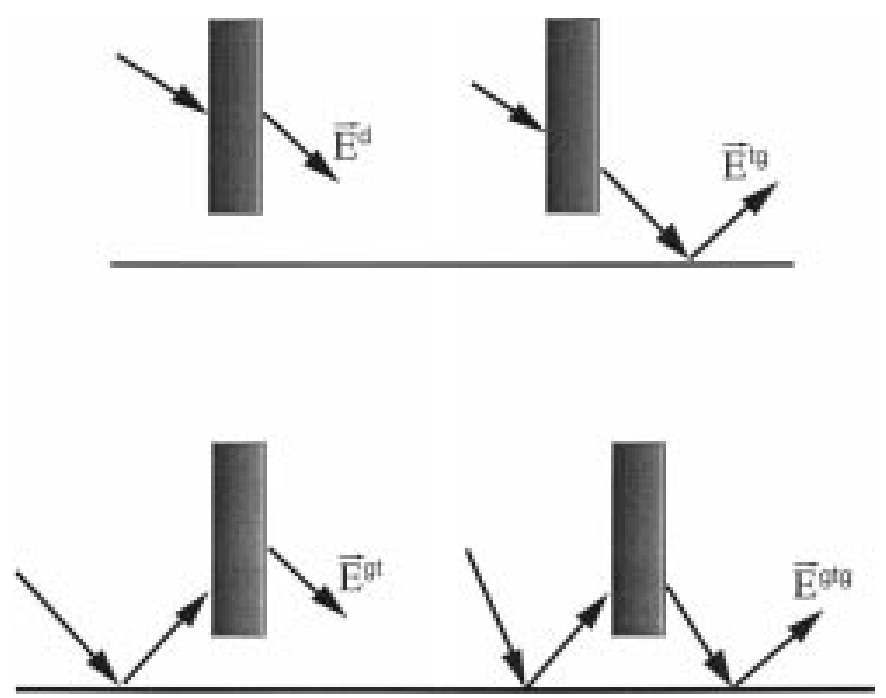

Fig. 3. Three geometrical optics (GO) components of the scattered field from a cylinder above the ground plane, along with forward scattered component

However, if the multiple scattering among branches and leaves is ignored, a single scattering model can be constructed. To a 


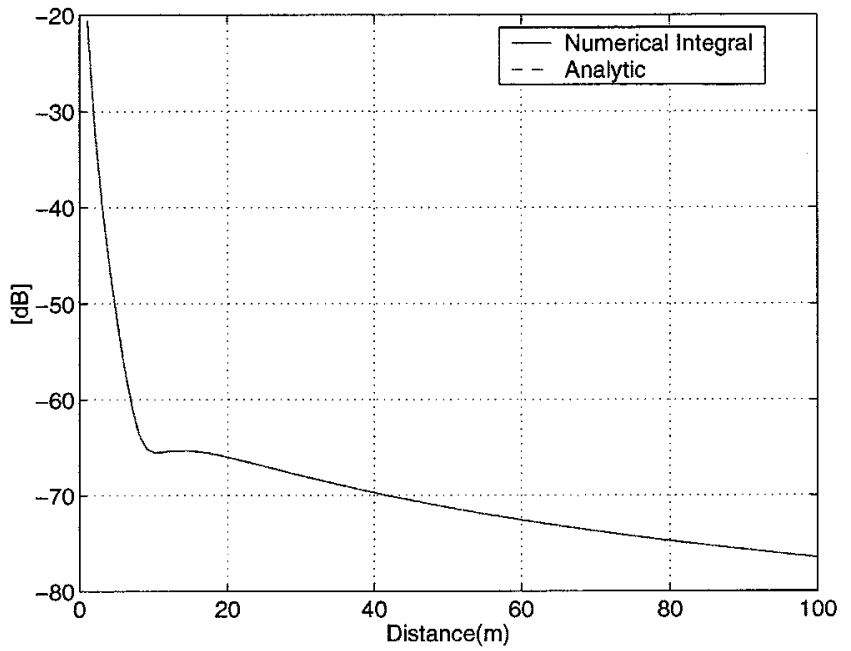

(a)

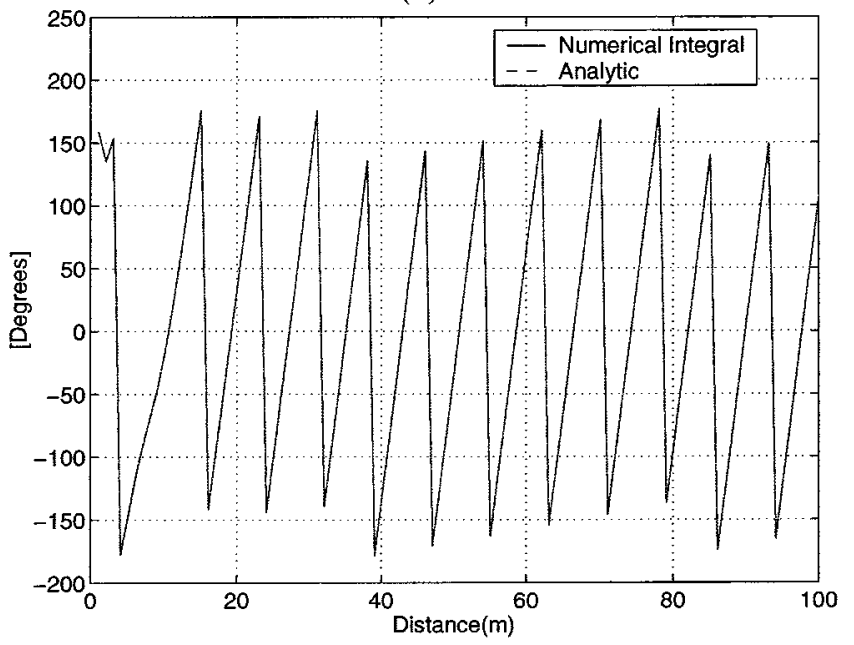

(b)

Fig. 4. Numerical and analytic evaluation of $\int_{0}^{L}\left(e^{j k_{0}\left(\left|\vec{r}-\vec{r}^{\prime}\right|-\cos \beta z^{\prime}\right)}\right) /(\mid \vec{r}-$ $\left.\overrightarrow{r^{\prime}} \mid\right) d z^{\prime}$. (a) Magnitude. (b) Phase.

high degree of accuracy this model can predict the first order statistics of the total field within a forest. This is because through the averaging process the dominant portion of the total field (coherent effect) can survive and the multiple interaction is minimized. In modeling the forest, one approach is to distribute the vegetation particles (leaves and branches) uniformly, however, it is found that for frequencies up to about $3 \mathrm{GHz}$ it is important to preserve the structural information of tree canopies for accurate prediction of radar backscatter [6]. This effect is expected to be even more important when the observation point is within the forest. Basically, not only the coherence of the scattered fields from the vegetation structure must be preserved, but also the near-field effects of lower branches and tree trunks near the observation point must be accounted for very accurately.

Fig. 1 shows the geometry of the communication problem where a receiver (or a transmitter) embedded in a foliage is communicating with a transmitter (or a receiver) above the foliage layer. As the signal passes through the foliage, it experiences attenuation and scattering which depend on signal parameters

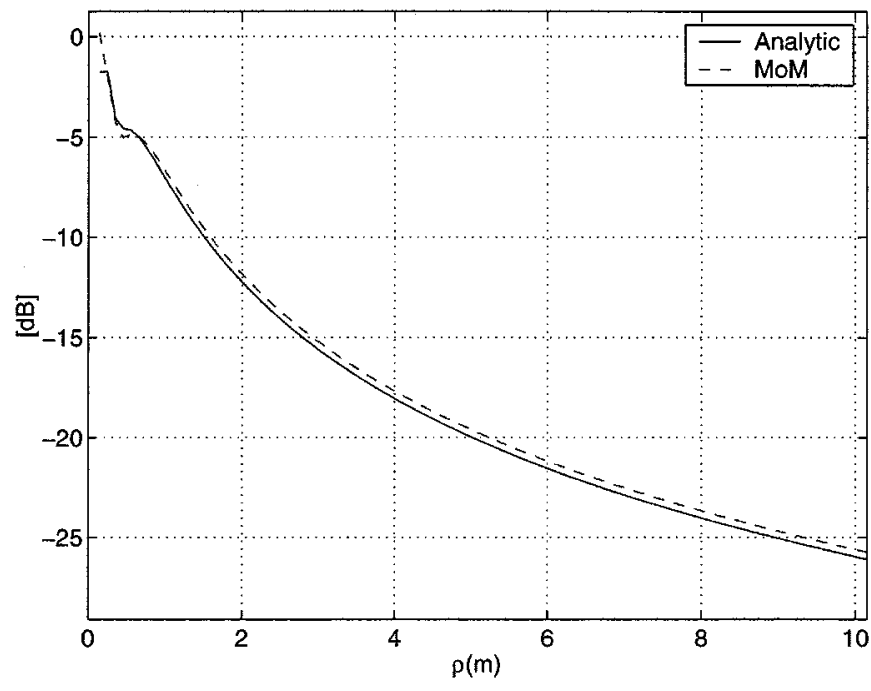

(a)

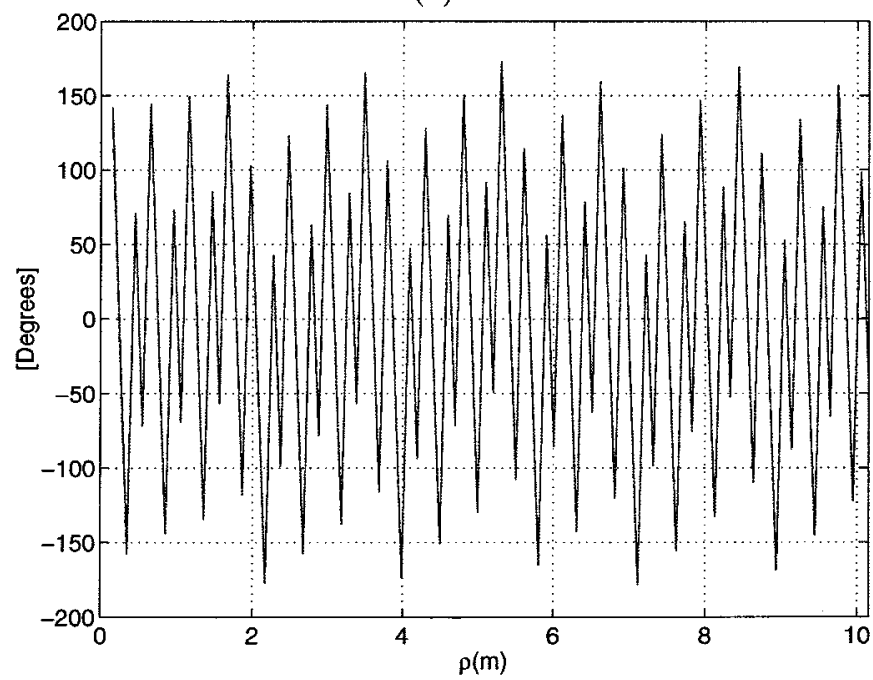

(b)

Fig. 5. Comparison of analytic and MoM results of scattered field $\left(E_{z}\right)$ from a finite cylinder whose radius is $5 \mathrm{~cm}$ and dielectric constant is $5+j$. The length of the finite cylinder is $4 \lambda$ and the observation line is $z=L / 2-\rho / \tan \beta$. TM wave is incident at $\beta=60^{\circ}$ and $\phi_{i}=180^{\circ}$. (a) Magnitude. (b) Phase.

such as frequency, polarization, and incidence angle, as well as vegetation attributes such as tree type, density, and height. As mentioned earlier, to model the interaction of electromagnetic waves with vegetation accurately, tree structures must be modeled rather accurately. Considering the number of branches and leaves on a tree and the variability in their sizes and orientations, generating a tree structure can be a very difficult task. This can be done efficiently by approximating tree structures by fractal geometries. Here we use a statistical Lindenmayer system [6] in conjunction with botanical properties pertinent to a specified tree specie. In this model, the geometry of vegetation particles are also approximated by canonical geometries such as dielectric disks, needles, and layered dielectric cylinders. Each particle in the medium is assumed to be illuminated by the incident 


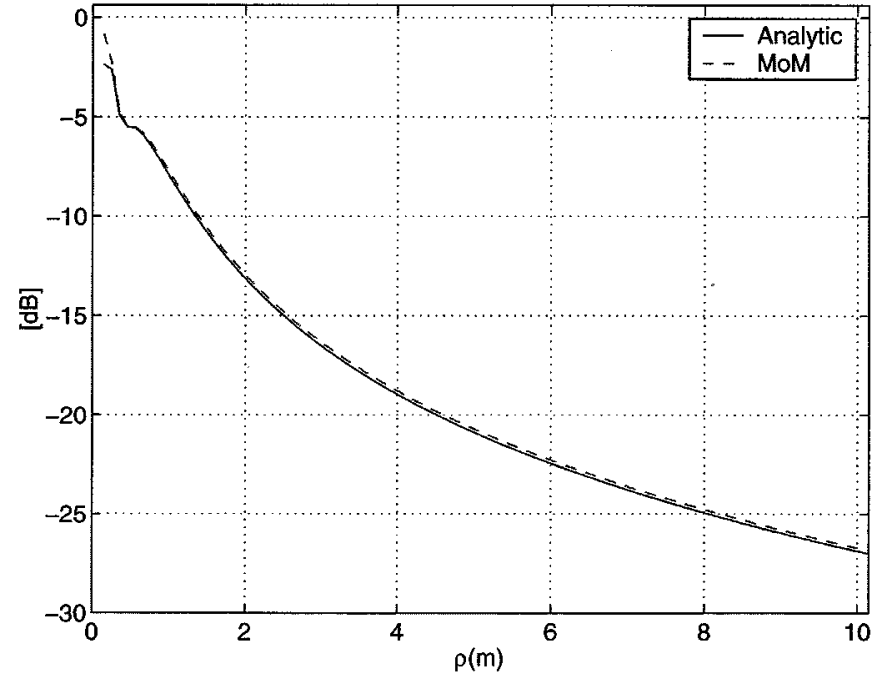

(a)

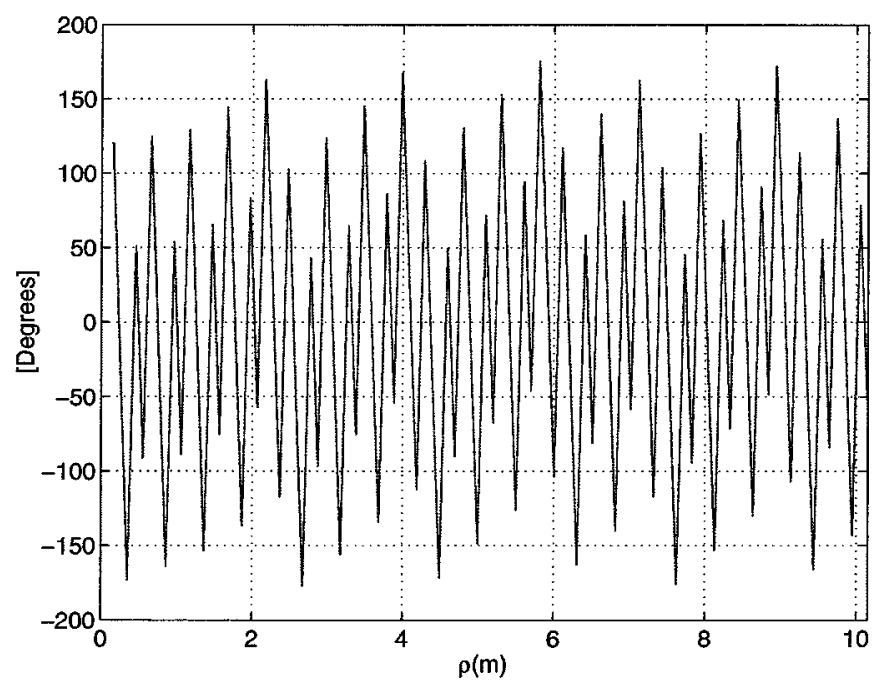

(b)

Fig. 6. Comparison of analytic and MoM results of scattered field $\left(E_{y}\right)$ from a finite cylinder whose radius is $5 \mathrm{~cm}$ and dielectric constant is $5+j$. The length of the finite cylinder is $4 \lambda$, and the observation line is $z=L / 2-\rho / \tan \beta$. TE wave is incident at $\beta=60^{\circ}$, and $\phi_{i}=180^{\circ}$. (a) Magnitude (b) Phase.

wave, attenuated by the foliage along the ray between the particle and the canopy top. The attenuation through the foliage is calculated using Foldy's approximation [12] and single scattering theory is invoked to compute the field scattered from all vegetation particles and their images in the ground plane in the vicinity of the observation point. The total field is then obtained by adding all scattered field components coherently.

\section{A. A Uniform Near-Field to Far-Field Expression for Scattering From Finite Dielectric Cylinders}

A building block for simulated fractal trees are finite dielectric cylinders which can be used to represent tree trunks, branches, and twigs. To compute the scattered field from a fractal tree, an analytic or numeric scattering solution is needed. Unfortunately, exact analytical solutions for finite dielectric cylinders do not exist. Numerical methods, of course, can

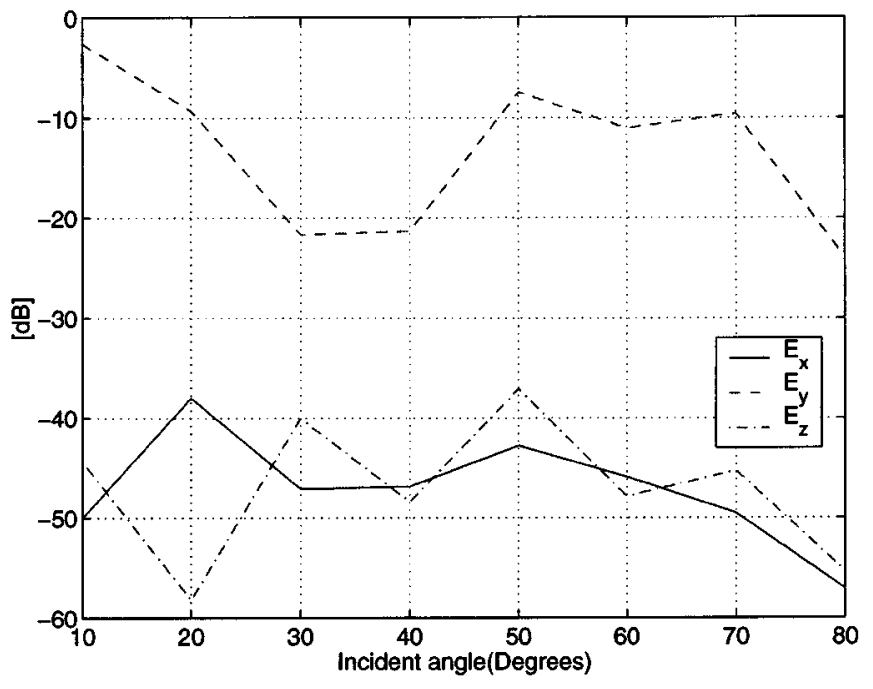

(a)

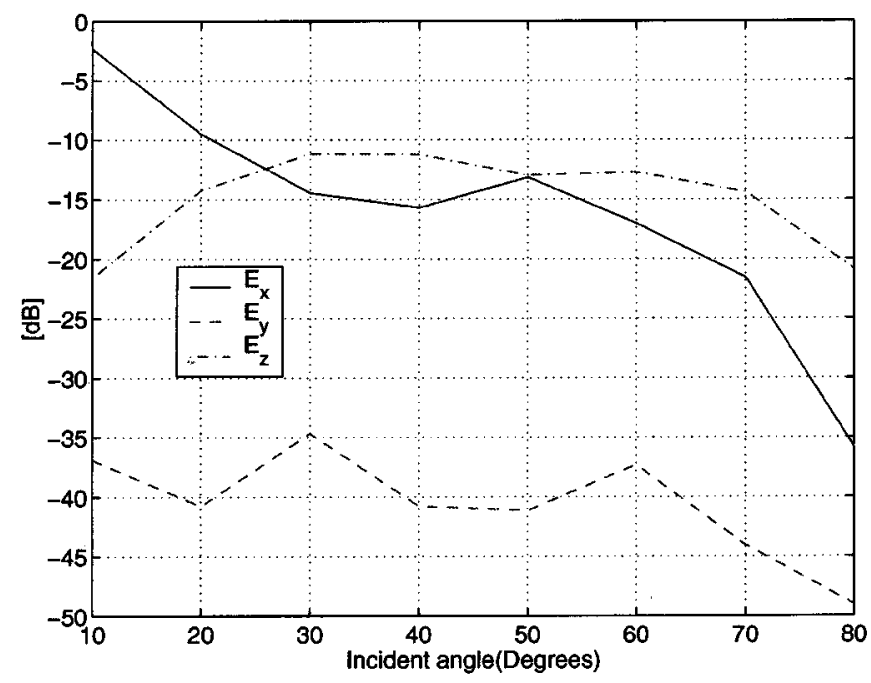

(b)

Fig. 7. Mean of total field at $z=1 \mathrm{~m}$ under pine trees as a function of incidence angle. The trees are located randomly with a density, $0.1 / \mathrm{m}^{2}$. (a) The incident wave is $h$-polarized. (b) The incident wave is $v$-polarized.

provide very accurate and general solutions for finite dielectric cylinders. However, considering the number of dielectric cylinders in a given tree and the fact that the length or the diameter of these cylinders may be comparable to or much larger than a wavelength, the computation time for the scattering solution becomes exorbitant. To circumvent this difficulty, an approximate analytical solution for the scattered field from a finite dielectric cylinder is formulated. For long cylinders (cylinders with large aspect ratio), significant scattered field exists only in a forward scattering cone [see Fig. 2(b)] [5]. Hence an accurate formulation for the scattered field in this region is of great interest. The formulation presented in this section provides very accurate results anywhere (from near field to far field) within and in the vicinity of the forward scattering region. This approximate solution is obtained by assuming that the equivalent surface electric $\left(\overrightarrow{\mathbf{J}}_{e}\right)$ and magnetic $\left(\overrightarrow{\mathbf{J}}_{m}\right)$ currents are the same as those of an infinite cylinder whose radius and dielectric constant are equal to those of the finite cylinder. It has been shown that approximate currents can provide accurate results in the far-field if the 


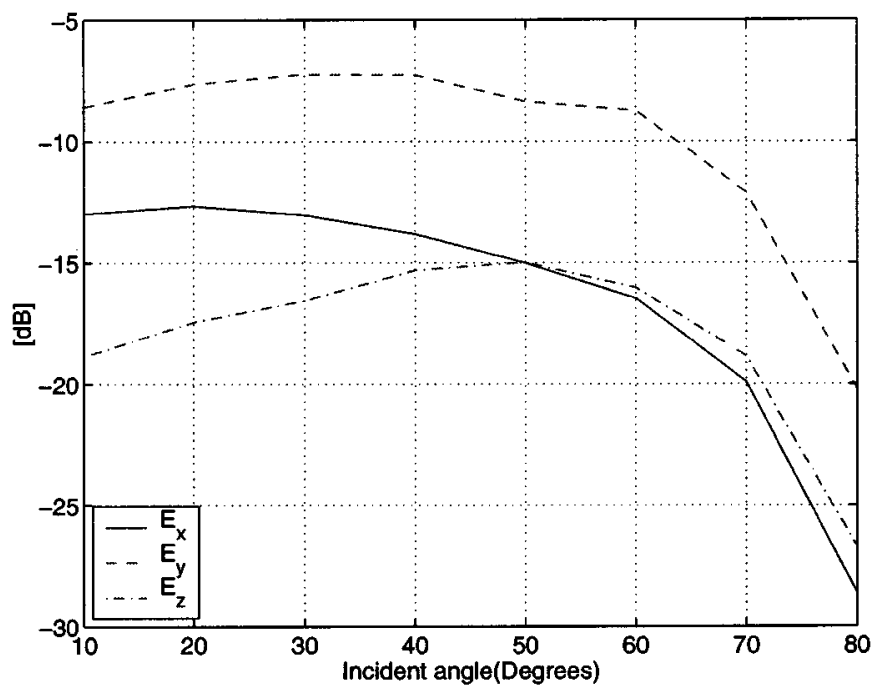

(a)

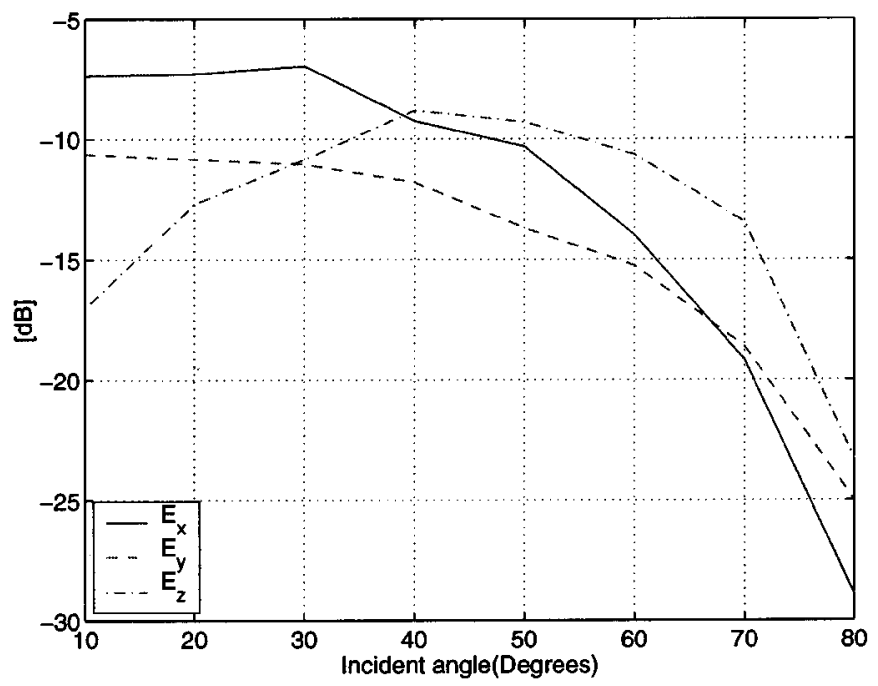

(b)

Fig. 8. Standard deviation of total field at $z=1 \mathrm{~m}$ under pine trees as a function of incidence angle. The trees are located randomly with a density, $0.1 / \mathrm{m}^{2}$. (a) The incident wave is $h$-polarized. (b) The incident wave is $v$-polarized.

length of the cylinder is large compared to both wavelength and cylinder radius $(L \gg \lambda$ and $a$ ), and the angle of incidence with respect to the cylinder axis is relatively large $\left(\beta \geq 10^{\circ}\right)$ [4], [5]. Analytic expressions for these currents are given by [4], [5]

$$
\begin{aligned}
\overrightarrow{\mathbf{J}}_{e}(\phi, z)= & \overrightarrow{\mathbf{J}}_{e}(\phi) e^{-j k_{0} z \cos \beta} \\
= & \sum_{m=-\infty}^{\infty}(-j)^{m} e^{j m \phi}\left[Q_{1 m} Y_{0}(\sin \phi \hat{x}\right. \\
& \left.-\cos \phi \hat{y})+Q_{2 m} \hat{z}\right] e^{-j k_{0} z \cos \beta} \\
\overrightarrow{\mathbf{J}}_{m}(\phi, z)= & \overrightarrow{\mathbf{J}}_{m}(\phi) e^{-j k_{0} z \cos \beta} \\
= & \sum_{m=-\infty}^{\infty}(-j)^{m} e^{j m \phi}\left[-\bar{Q}_{1 m}(\sin \phi \hat{x}\right. \\
& \left.-\cos \phi \hat{y})+\bar{Q}_{2 m} \hat{z}\right] e^{-j k_{0} z \cos \beta}
\end{aligned}
$$

where $k_{0}$ is the free-space propagation constant, and $Y_{0}=1 / Z_{0}$ is the free-space admittance. The terms, $Q_{1 m}, Q_{2 m}, \bar{Q}_{1 m}$, and $\bar{Q}_{2 m}$ are given by

$$
\begin{aligned}
Q_{1 m}= & h_{z} J_{m}\left(x_{0}\right)+B_{m} H_{m}\left(x_{0}\right) \\
Q_{2 m}= & -\frac{Y_{0}}{j k_{0} \sin ^{2} \beta}\left\{k_{0} \sin \beta\left[e_{z} J_{m}^{\prime}\left(x_{0}\right)+A_{m} H_{m}^{\prime}\left(x_{0}\right)\right]\right. \\
& \left.+\frac{j m \cos \beta}{a}\left[h_{z} J_{m}\left(x_{0}\right)+B_{m} H_{m}\left(x_{0}\right)\right]\right\} \\
\bar{Q}_{1 m}= & e_{z} J_{m}\left(x_{0}\right)+A_{m} H_{m}\left(x_{0}\right) \\
\bar{Q}_{2 m}= & -\frac{1}{j k_{0} \sin ^{2} \beta}\left\{k_{0} \sin \beta\left[h_{z} J_{m}^{\prime}\left(x_{0}\right)+B_{m} H_{m}^{\prime}\left(x_{0}\right)\right]\right. \\
& \left.-\frac{j m \cos \beta}{a}\left[e_{z} J_{m}\left(x_{0}\right)+A_{m} H_{m}\left(x_{0}\right)\right]\right\}
\end{aligned}
$$

where $x_{0}=k_{0} a \sin \beta, e_{z}=\overrightarrow{\mathbf{E}}^{i} \cdot \hat{z}, h_{z}=Z_{0} \overrightarrow{\mathbf{H}}^{i} \cdot \hat{z}, J_{m}$, and $H_{m}$ are the $m$ th-order Bessel and Hankel function of the first kind, respectively, and the ' indicates the derivative with respect to the entire argument. Other terms, such as $A_{m}$, and $B_{m}$ are given in [4], [5]. Using these currents, the electric field can be written in terms of electric $\left(\overrightarrow{\boldsymbol{\Pi}}_{e}\right)$, and magnetic $\left(\overrightarrow{\boldsymbol{\Pi}}_{m}\right)$ Hertz potentials using

$$
\overrightarrow{\mathbf{E}}(\vec{r})=\nabla \times \nabla \times \overrightarrow{\boldsymbol{\Pi}}_{e}(\vec{r})+j k_{0} Z_{0} \nabla \times \overrightarrow{\boldsymbol{\Pi}}_{m}(\vec{r})
$$

where

$$
\begin{aligned}
\overrightarrow{\boldsymbol{\Pi}}_{e} & =\frac{j Z_{0}}{4 \pi k_{0}} \int_{0}^{2 \pi} \overrightarrow{\mathbf{J}}_{e}\left(\phi^{\prime}\right) \int_{0}^{L} \frac{e^{j k_{0}\left(\left|\vec{r}-\overrightarrow{r^{\prime}}\right|-\cos \beta z^{\prime}\right)}}{\left|\vec{r}-\overrightarrow{r^{\prime}}\right|} a d z^{\prime} d \phi^{\prime} \\
\overrightarrow{\boldsymbol{\Pi}}_{m} & =\frac{j Y_{0}}{4 \pi k_{0}} \int_{0}^{2 \pi} \overrightarrow{\mathbf{J}}_{m}\left(\phi^{\prime}\right) \int_{0}^{L} \frac{e^{j k_{0}\left(\left|\vec{r}-\overrightarrow{r^{\prime}}\right|-\cos \beta z^{\prime}\right)}}{\left|\vec{r}-\overrightarrow{r^{\prime}}\right|} a d z^{\prime} d \phi^{\prime}
\end{aligned}
$$

In situations where the observation point is not in the far-field region of the cylinder, a difficulty arises from the evaluation of $\int_{0}^{L}\left(e^{j k_{0}\left(\left|\vec{r}-\vec{r}^{\prime}\right|-\cos \beta z^{\prime}\right)}\right) /\left(\left|\vec{r}-\overrightarrow{r^{\prime}}\right|\right) d z^{\prime}$. As will be shown, using an integral transformation, this integral can be represented by an approximate closed-form formula which retains a high degree of accuracy. Using the identity, $\left(e^{j k_{0}\left|\vec{r}-\vec{r}^{\prime}\right|}\right) /\left(\left|\vec{r}-\overrightarrow{r^{\prime}}\right|\right)=$ $(j / 2) \int_{-\infty}^{\infty} H_{0}^{(1)}\left(k_{1} \zeta\right) e^{j \alpha_{1}\left(z-z^{\prime}\right)} d \alpha_{1}[13]$, the integral is transformed to a more appropriate form

$$
\begin{aligned}
I= & \int_{0}^{L} \frac{e^{j k_{0}\left(\left|\vec{r}-\overrightarrow{r^{\prime}}\right|-\cos \beta z^{\prime}\right)}}{\left|\vec{r}-\overrightarrow{r^{\prime}}\right|} d z^{\prime} \\
= & \frac{j}{2} \int_{0}^{L} \int_{-\infty}^{\infty} H_{0}\left(k_{1} \zeta\right) e^{j \alpha_{1}\left(z-z^{\prime}\right)} d \alpha_{1} \cdot e^{-j \alpha z^{\prime}} d z^{\prime} \\
= & \frac{1}{2} \int_{-\infty}^{\infty} \frac{H_{0}\left(k_{1} \zeta\right)}{\alpha_{1}+\alpha} e^{j \alpha_{1} z} d \alpha_{1} \\
& -e^{-j \alpha L} \frac{1}{2} \int_{-\infty}^{\infty} \frac{H_{0}\left(k_{1} \zeta\right)}{\alpha_{1}+\alpha} e^{j \alpha_{1}(z-L)} d \alpha_{1}
\end{aligned}
$$

where $\zeta=\sqrt{\left(x-x^{\prime}\right)^{2}+\left(y-y^{\prime}\right)^{2}}$, and $\alpha=k_{0} \cos \beta$, and $k_{1}^{2}=k_{0}^{2}-\alpha_{1}^{2}$. Each integral in the above equation can be trans- 
formed by following the standard steepest decent method procedure [13], [14] into

$$
\begin{aligned}
I_{i}= & \int_{-\infty}^{\infty} \frac{H_{0}\left(k_{1} \zeta\right)}{\alpha_{1}+\alpha} e^{j \alpha_{1} z_{i}} d \alpha_{1} \\
\sim & \sqrt{\frac{2}{\pi k_{0} \zeta}} e^{-j \pi / 4} \cdot e^{j k_{0} r_{i}} \\
& \times \int_{-\infty}^{\infty} \frac{\sqrt{\cos w}}{\sin w+\cos \beta} e^{-k_{0} r_{i} s^{2}} \frac{d w}{d s} d s
\end{aligned}
$$

where $i$ can be 1 or $2, z_{1}=z$, and $z_{2}=z-L$. Also $(d w / d s)=(2 \sqrt{-j}) /\left(\sqrt{1+\cos \left(w-\theta_{s}\right)}\right), \cos \theta_{s}=$ $\left(\zeta / r_{i}\right), r_{i}=\sqrt{\zeta^{2}+z_{i}^{2}}$, and $\cos \left(w-\theta_{s}\right)=1+j s^{2}$. Since the resulting integrand has a singularity at $w=\beta-\pi / 2$ the integrand must be decomposed into two parts: one that contains the singularity and the other one which is regular, that is

$G(s)=\frac{\sqrt{\cos w}}{\sin w+\cos \beta} \cdot \frac{2 \sqrt{-j}}{\sqrt{1+\cos \left(w-\theta_{s}\right)}}=\frac{b}{s-s_{1}}+T(s)$

where $s_{1}= \pm \sqrt{j\left[1-\sin \left(\beta-\theta_{s}\right)\right]}$ and $b$ is the residue of $G(s)$ at the singularity which is given by

$$
\begin{aligned}
b & =\lim _{s \rightarrow s_{1}}\left(s-s_{1}\right) G(s)=\frac{1}{\sqrt{\sin \beta}} \\
T(0) & =G(0)+\frac{b}{s_{1}} \\
& =e^{-j \pi / 4}\left[\frac{\sqrt{2 \cos \theta_{s}}}{\sqrt{\sin \theta_{s}+\cos \beta}} \pm \frac{1}{\sqrt{\sin \beta} \sqrt{1-\sin \left(\beta-\theta_{s}\right)}}\right] .
\end{aligned}
$$

Here the $+\operatorname{sign}$ is used for $\theta_{s}<\beta-\pi / 2$, the - sign is used for $\theta_{s}>\beta-\pi / 2$, and $T(0)=$ $\left(e^{-j \pi / 4}\right) /(\sqrt{\sin \beta})[(\cos \beta) /(2 \sin \beta)-\sqrt{2 \sin \beta}]$ for $\theta_{s}=\beta-\pi / 2$. Noting that

$$
\begin{aligned}
\int_{-\infty}^{\infty} \frac{b}{s-s_{1}} e^{-k_{0} r s^{2}} d s+\int_{-\infty}^{\infty} T(s) e^{-k_{0} r s^{2}} d s \\
\quad=j \pi b e^{-k_{0} r s_{1}^{2}}\left[\operatorname{erf}\left(j \sqrt{k_{0} r} s_{1}\right)+\Lambda\right]+T(0) \sqrt{\frac{\pi}{k_{0} r}}
\end{aligned}
$$

where

$$
\Lambda= \begin{cases}1 & \operatorname{Im}\left[s_{1}\right]>0 \\ 0 & \operatorname{Im}\left[s_{1}\right]=0 \\ -1 & \operatorname{Im}\left[s_{1}\right]<0\end{cases}
$$

the final expressions for $I_{1}$ and $I_{2}$ can be written as

$$
\begin{aligned}
I_{i} \sim e^{-j \pi / 4}\left[\frac{e^{j k_{0} r_{i}}}{k_{0}} \sqrt{\frac{2}{\zeta r_{i}}} T(0)\right. \\
\left.\quad+j \sqrt{\frac{2 \pi}{k_{0} \sin \beta \zeta}} e^{j k_{0} r_{i} \sin \left(\beta-\theta_{s}\right)} \cdot\left[\operatorname{erf}\left(j \sqrt{k_{0} r_{i}} s_{1}\right)+\Lambda\right]\right]
\end{aligned}
$$

where $\operatorname{erf}(\cdot)$ is the error function [13]-[15]. Noting that for large $k_{0} \sin \beta \zeta$ the quantity $\sqrt{(2 \pi) /\left(k_{0} \sin \beta \zeta\right)} e^{j k_{0} r_{i} \sin \left(\beta-\theta_{s}\right)}$ $\pi H_{0}\left(k_{0} \sin \beta \zeta\right) e^{-j k_{0} \cos \beta z_{i}}, \quad$ a $\quad$ better approximation for $I_{i}$ may be written as

$$
\begin{aligned}
I_{i} \sim \frac{e^{j k_{0} r_{i}}}{k_{0}} \sqrt{\frac{2}{\zeta r_{i}}} T(0) e^{-j \pi / 4}+j \pi H_{0}\left(k_{0} \sin \beta \zeta\right) \\
\times\left[\operatorname{erf}\left(j \sqrt{k_{0} r} s_{1}\right)+\Lambda\right] e^{-j k_{0} \cos \beta z_{i}} .
\end{aligned}
$$

Hence the expression for the electric Hertz potential in (4), can be written as

$$
\begin{aligned}
\overrightarrow{\mathbf{\Pi}}_{e} \sim & \frac{j Z_{0} a}{8 \pi k_{0}} \sum_{m=-\infty}^{\infty}(-j)^{m} \int_{0}^{2 \pi} e^{j m \phi^{\prime}}\left[Q _ { 1 m } Y _ { 0 } \left(\sin \phi^{\prime} \hat{x}\right.\right. \\
& \left.\left.-\cos \phi^{\prime} \hat{y}\right)+Q_{2 m} \hat{z}\right] \cdot\left[I_{1}-I_{2} e^{-j k_{0} \cos \beta L}\right] d \phi^{\prime} .
\end{aligned}
$$

It is not obvious how to carry out the above integral with respect to $\phi^{\prime}$ analytically, however if $r^{2}+a^{2} \gg a \rho$ is assumed, an analytical expression can be obtained using the following approximations:

$$
\begin{aligned}
r_{1} & =\sqrt{\left(x-x^{\prime}\right)^{2}+\left(y-y^{\prime}\right)^{2}+z^{2}} \\
& \approx \sqrt{r^{2}+a^{2}}-\frac{\rho a \cos \left(\phi-\phi^{\prime}\right)}{\sqrt{r^{2}+a^{2}}} \\
\sqrt{r_{1}} s_{1} & \approx \sqrt{r_{a 1}} s_{1}
\end{aligned}
$$

where $r=\sqrt{x^{2}+y^{2}+z^{2}}$, and $\rho=\sqrt{x^{2}+y^{2}}$. Similarly

$$
\begin{aligned}
r_{2} & =\sqrt{\left(x-x^{\prime}\right)^{2}+\left(y-y^{\prime}\right)^{2}+(z-L)^{2}} \\
& \approx \sqrt{r_{L}^{2}+a^{2}}-\frac{\rho a \cos \left(\phi-\phi^{\prime}\right)}{\sqrt{r_{L}^{2}+a^{2}}} \\
\sqrt{r_{2}} s_{1} & \approx \sqrt{r_{a 2}} s_{1}
\end{aligned}
$$

where $r_{L}=\sqrt{x^{2}+y^{2}+(z-L)^{2}}$. Therefore, with the aid of the integral relations given in [4], [5], the two integrals in (7) can be evaluated analytically as

$$
\begin{aligned}
\vec{\pi}_{e}^{i}= & \frac{j Z_{0} a}{4 \pi k_{0}} \sum_{m=-\infty}^{\infty}(-j)^{m} \int_{0}^{2 \pi} e^{j m \phi^{\prime}}\left[Q _ { 1 m } Y _ { 0 } \left(\sin \phi^{\prime} \hat{x}\right.\right. \\
& \left.\left.-\cos \phi^{\prime} \hat{y}\right)+Q_{2 m} \hat{z}\right] I_{i} d \phi^{\prime} \\
& \sim \frac{j Z_{0} a}{2 k_{0}}\left[e^{-j \pi / 4} \frac{e^{j k_{0} r_{a i}}}{k_{0}} \sqrt{\frac{2}{\rho r_{a i}}} T(0)\right. \\
& \times \sum_{m=-\infty}^{\infty}(-1)^{m} \vec{C}_{m}\left(\frac{\rho a}{r_{a i}}\right) e^{j m \phi} \\
& +j \pi H_{0}\left(k_{0} \sin \beta \rho_{a}\right)\left[\operatorname{erf}\left(j \sqrt{k_{0} r_{a i}} s_{1}\right)+\Lambda\right] e^{-j k_{0} \cos \beta z} \\
& \left.\cdot \sum_{m=-\infty}^{\infty}(-1)^{m} \vec{C}_{m}\left(\frac{\rho a}{r_{a i}} \sin \beta\right) e^{j m \phi}\right]
\end{aligned}
$$

where

$$
\begin{aligned}
r_{a 1}= & \sqrt{r^{2}+a^{2}}, \quad r_{a 2}=\sqrt{r_{L}^{2}+a^{2}} \\
\vec{C}_{m}\left(y_{0}\right)= & Y_{0} Q_{1 m} P_{1 m}\left(y_{0}\right) \hat{x}-Y_{0} Q_{1 m} P_{2 m}\left(y_{0}\right) \hat{y} \\
& +Q_{2 m} J_{m}\left(y_{0}\right) \hat{z} \\
P_{1 m}\left(y_{0}\right)= & j \sin \phi J_{m}^{\prime}\left(y_{0}\right)-\cos \phi \frac{m}{y_{0}} J_{m}\left(y_{0}\right) \\
P_{2 m}\left(y_{0}\right)= & -j \cos \phi J_{m}^{\prime}\left(y_{0}\right)+\sin \phi \frac{m}{y_{0}} J_{m}\left(y_{0}\right) .
\end{aligned}
$$


Similarly

$$
\begin{aligned}
\vec{\pi}_{m}^{i} \sim & \frac{j Y_{0} a}{2 k_{0}}\left[e^{-j \pi / 4} \frac{e^{j k_{0} r_{a i}}}{k_{0}} \sqrt{\frac{2}{\rho r_{a i}}} T(0)\right. \\
& \times \sum_{m=-\infty}^{\infty}(-1)^{m} \vec{D}_{m}\left(\frac{\rho a}{r_{a i}}\right) e^{j m \phi} \\
& +j \pi H_{0}\left(k_{0} \sin \beta \rho_{a}\right)\left[\operatorname{erf}\left(j \sqrt{k_{0} r_{a i}} s_{1}\right)+\Lambda\right] \\
& \left.\times e^{-j k_{0} \cos \beta z} \cdot \sum_{m=-\infty}^{\infty}(-1)^{m} \vec{D}_{m}\left(\frac{\rho a}{r_{a i}} \sin \beta\right) e^{j m \phi}\right]
\end{aligned}
$$

where $\vec{D}_{m}\left(y_{0}\right)=-\bar{Q}_{1 m} P_{1 m}\left(y_{0}\right) \hat{x}+\bar{Q}_{1 m} P_{2 m}\left(y_{0}\right) \hat{y}+$ $\bar{Q}_{2 m} J_{m}\left(y_{0}\right) \hat{z}$. Finally, the two Hertz vectors can be expressed by

$$
\begin{aligned}
\overrightarrow{\boldsymbol{\Pi}}_{e} & \sim \frac{1}{2}\left(\overrightarrow{\boldsymbol{\pi}}_{e}^{1}-\overrightarrow{\boldsymbol{\pi}}_{e}^{2} e^{-j k_{0} \cos \beta L}\right) \\
\overrightarrow{\boldsymbol{\Pi}}_{m} & \sim \frac{1}{2}\left(\overrightarrow{\boldsymbol{\pi}}_{m}^{1}-\overrightarrow{\boldsymbol{\pi}}_{m}^{2} e^{-j k_{0} \cos \beta L}\right) .
\end{aligned}
$$

According to (3), the scattered electric field can be evaluated by taking the curl of the approximate closed form expressions of the Hertz potentials. Noting that the observation point is in the far field with regard to cylinder diameter $\left(\rho>\left(8 a^{2} / \lambda\right)\right)$ the far-field approximation for the $\nabla$ operator is expressed as

$$
\begin{aligned}
\nabla e^{j k_{0} r_{a 1}} & =j k_{0} \frac{\vec{r}_{a 1}}{r_{a 1}} e^{j k_{0} r_{a 1}} \\
\nabla e^{j k_{0} r_{a 2}} & =j k_{0} \frac{\vec{r}_{a 2}}{r_{a 2}} e^{j k_{0} r_{a 2}} \\
\nabla e^{j k_{0}\left(\sin \beta \rho_{a}-\cos \beta z\right)} & =j k_{0} \hat{k}_{s} e^{j k_{0}\left(\sin \beta \rho_{a}-\cos \beta z\right)}
\end{aligned}
$$

where $\hat{k}_{s}=\sin \beta \cos \phi \hat{x}+\sin \beta \sin \phi \hat{y}-\cos \beta \hat{z}$. After some algebraic manipulation, the complete expression of the electric field for a finite cylinder may be written as

$$
\begin{aligned}
\overrightarrow{\mathbf{E}} \sim & -j \frac{k_{0} a}{4}\left[e^{-j \pi / 4} \frac{e^{j k_{0} r_{a 1}}}{k_{0}} \sqrt{\frac{2}{\rho r_{a 1}}} T(0) \sum_{m=-\infty}^{\infty}(-1)^{m} e^{j m \phi}\right. \\
& \times\left[Z_{0} \hat{r}_{a 1} \times \hat{r}_{a 1} \times \vec{C}_{m}\left(\frac{\rho a}{r_{a 1}}\right)+\hat{r}_{a 1} \times \vec{D}_{m}\left(\frac{\rho a}{r_{a 1}}\right)\right] \\
& -e^{-j k_{0} \cos \beta L} e^{-j \pi / 4} \frac{e^{j k_{0} r_{a 2}}}{k_{0}} \sqrt{\frac{2}{\rho r_{a 2}}} T(0) \\
& +\sum_{m=-\infty}^{\infty}(-1)^{m} e^{j m \phi}\left[Z_{0} \hat{r}_{a 2} \times \hat{r}_{a 2} \times \vec{C}_{m}\left(\frac{\rho a}{r_{a 2}}\right)\right. \\
& \left.+\hat{r}_{a 2} \times \vec{D}_{m}\left(\frac{\rho a}{r_{a 2}}\right)\right]+j \pi H_{0}\left(k_{0} \sin \beta \rho_{a}\right) e^{-j k_{0} \cos \beta z} \\
& \times \sum_{m=-\infty}^{\infty}(-1)^{m} e^{j m \phi}\left[Z_{0} \hat{k}_{s} \times \hat{k}_{s} \times \vec{C}_{m}\left(\frac{\rho a}{\rho_{a}} \sin \beta\right)\right. \\
& \left.+\hat{k}_{s} \times \vec{D}_{m}\left(\frac{\rho a}{\rho_{a}} \sin \beta\right)\right]\left[\operatorname{erf}\left(j \sqrt{k_{0} r_{a 1}} s_{1}\right)\right. \\
& \left.\left.+\Lambda_{1}-\operatorname{erf}\left(j \sqrt{k_{0} r_{a 2}} s_{1}\right)-\Lambda_{2}\right]\right] .
\end{aligned}
$$

Equation (10) is valid for observation points on or near the forward scattering cone. If the observation point is outside the cone (the effect of the pole can be neglected), another equation for the scattered field can be formulated. Using the asymptotic behavior of the error function, $\operatorname{erf}(z) \sim 1-\left(e^{-z^{2}}\right) /(\sqrt{\pi} z)$ [15], (6) can be reduced to

$$
I_{i} \sim-j \frac{e^{j k_{0} r_{i}}}{r_{i}} \frac{2}{k_{0}\left(\sin \theta_{s}+\cos \beta\right)} .
$$

Through the same procedure and approximations, a formulation for the scattered field that is valid outside the forward scattering cone can be written as

$$
\begin{aligned}
\overrightarrow{\mathbf{E}} \sim & -\frac{a}{2}\left[\frac{e^{j k_{0} r_{a 1}}}{r_{a 1}} \frac{1}{\sin \theta_{s}+\cos \beta} \sum_{m=-\infty}^{\infty}(-1)^{m} e^{j m \phi}\right. \\
& \times\left[Z_{0} \hat{r}_{a 1} \times \hat{r}_{a 1} \times \vec{C}_{m}\left(\frac{\rho a}{r_{a 1}}\right)+\hat{r}_{a 1} \times \vec{D}_{m}\left(\frac{\rho a}{r_{a 1}}\right)\right] \\
& -\frac{e^{j k_{0} r_{a 2}}}{r_{a 2}} \frac{e^{-j k_{0} \cos \beta L}}{\sin \theta_{s}+\cos \beta} \cdot \sum_{m=-\infty}^{\infty}(-1)^{m} e^{j m \phi} \\
& \times\left[Z_{0} \hat{r}_{a 2} \times \hat{r}_{a 2} \times \vec{C}_{m}\left(\frac{\rho a}{r_{a 2}}\right)+\hat{r}_{a 2} \times \vec{D}_{m}\left(\frac{\rho a}{r_{a 2}}\right)\right] .
\end{aligned}
$$

It is worth mentioning that (10) and (11) can be reduced to the formula derived in [5] for observation points in the near-field region and a formula given in [4] for observation points in the far-field region. To apply this formulation to a cylinder with arbitrary orientation, it is needed to transform the components of the incident wave in the global coordinate system $(X, Y, Z)$, to the local cylinder coordinate system $\left(x^{\prime}, y^{\prime}, z^{\prime}\right)$ where $z^{\prime}$-axis is parallel to the cylinder axis. After calculating the scattered field components in the local coordinates, they are transformed back to the global coordinate system. This procedure is mathematically expressed by

$$
\begin{aligned}
& \overrightarrow{\mathbf{E}}_{L}^{i}=U^{-1} \overrightarrow{\mathbf{E}}_{G}^{i} \\
& \overrightarrow{\mathbf{E}}_{G}^{s}=U \overrightarrow{\mathbf{E}}_{L}^{s}
\end{aligned}
$$

where $U$ is the transformation matrix and is given by

$$
U=\left(\begin{array}{ccc}
\cos \theta_{c} \cos \phi_{c} & -\sin \phi_{c} & \cos \phi_{c} \cos \phi_{c} \\
\cos \theta_{c} \sin \phi_{c} & \cos \phi_{c} & \sin \theta_{c} \sin \phi_{c} \\
-\sin \theta_{c} & 0 & \cos \theta_{c}
\end{array}\right)
$$

for a cylinder with orientation angles $\left(\theta_{c}, \phi_{c}\right)$, as is seen in Fig. 2.

\section{B. Scattering From Leaves and Needles}

The thickness of most broad leaf vegetation $(\tau \sim 0.1-0.5$ $\mathrm{mm}$ ) is very small compared to the wavelength at frequencies up to $X$ band. For these leaves the Rayleigh-Gans approximation [16] is used, which is valid, independent of the leaf surface dimensions relative to the wavelength. That is so long as the electric thickness of the leaf is very small, the Rayleigh-Gans approximation provides accurate results. For leaves where surface dimensions are large compared to the wavelength and their electric thickness becomes compared to the wavelength, a physical optic approximation [4] is used. This solution is valid for frequencies at $X$ band and above. A formulation for thin cylinders of arbitrary cross section [4] is used to model the scattering from vegetation needles. 


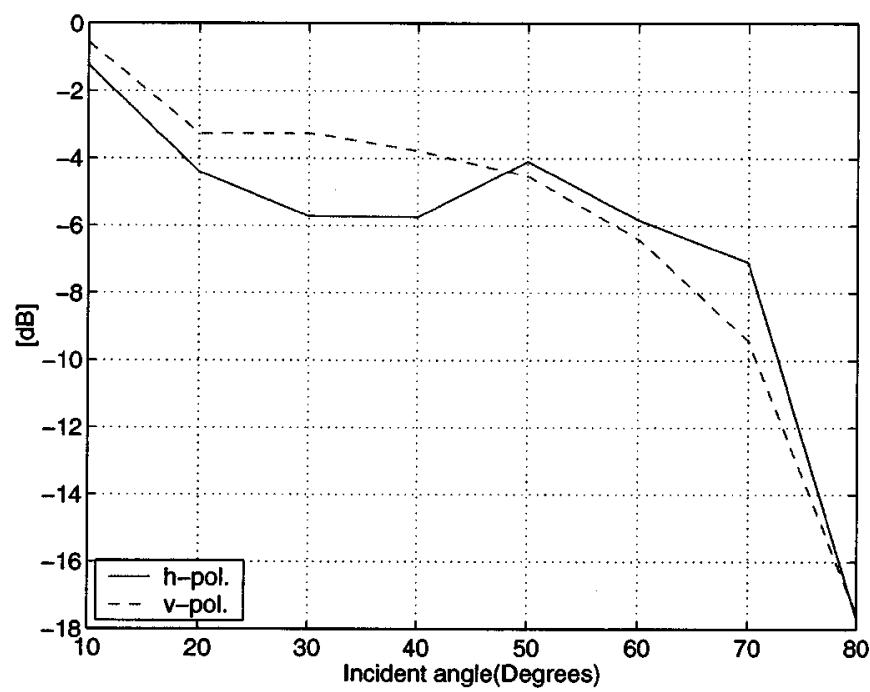

(a)

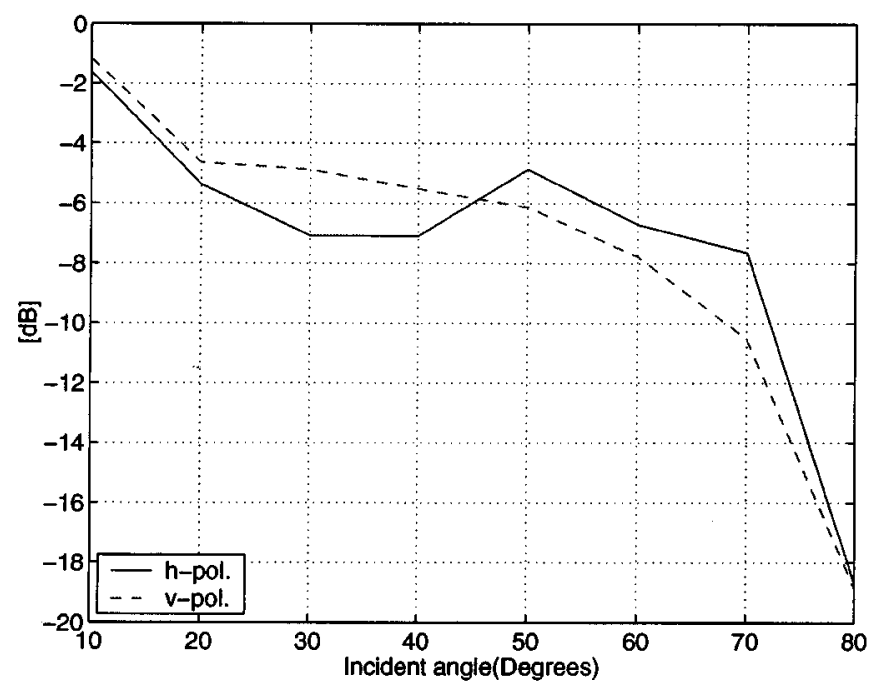

(b)

Fig. 9. Total and received power at $z=1 \mathrm{~m}$ under pine trees as a function of incidence angle. The trees are located randomly with a density, $0.1 / \mathrm{m}^{2}$. (a) $\left\langle|E|^{2}\right\rangle$. (b) Path loss.

\section{Effect of Ground Plane}

To calculate the scattered field accurately, the effect of the ground plane should be included into the final solution. The underlying soil surface is modeled by a half-space dielectric medium. However, to account for the exact effect of the halfspace dielectric using the Sommerfeld integral is very complicated and not necessary for the required degree of accuracy. Thus in this paper, only the three dominant GO components are incorporated into the final solution. The first component is the scattered field generated by the reflected incident field from the ground plane, denoted by $\overrightarrow{\mathbf{E}}^{g t}$, the second term is the scattered field reflected from the ground plane $\left(\overrightarrow{\mathbf{E}}^{t g}\right)$, and the third component is the scattered field generated by reflected incident field which is again reflected from the ground $\left(\overrightarrow{\mathbf{E}}^{g t g}\right)$ (see Fig. 3). These terms should be added coherently to the direct scattered field. The direction of the reflected incident wave is

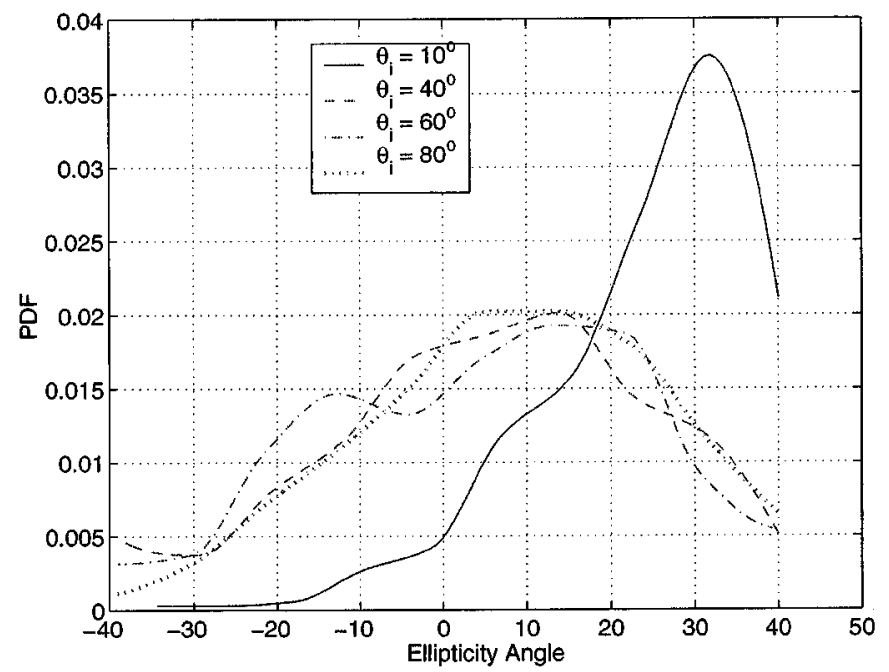

(a)

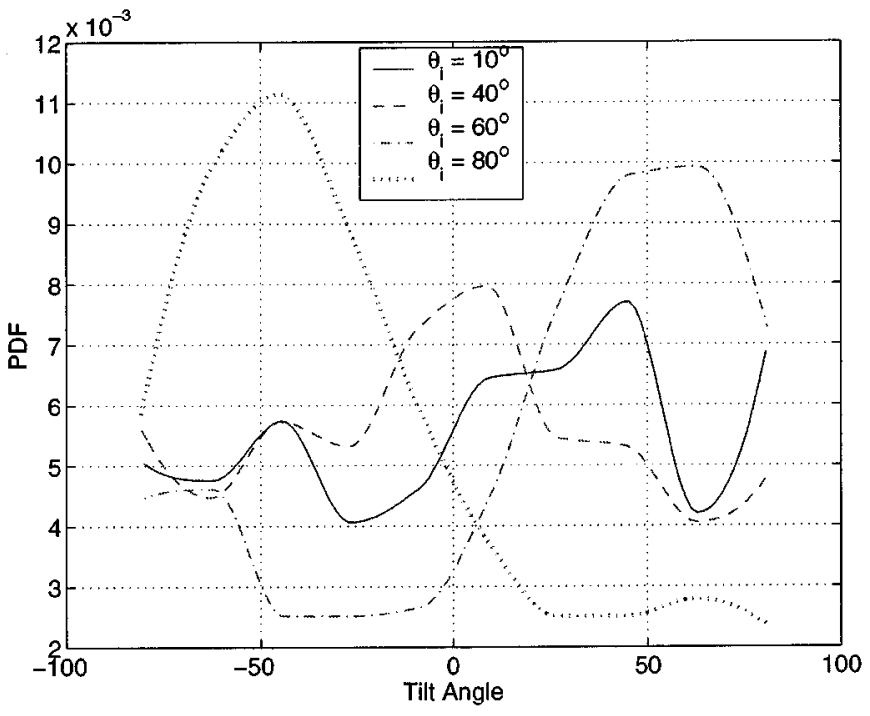

(b)

Fig. 10. pdf of polarization of the received signal at $z=1 \mathrm{~m}$ under pine trees. Four results are plotted whose incidence angles are $10^{\circ}, 40^{\circ}, 60^{\circ}$, and $80^{\circ}$. (a) PDF of ellipticity angle. (b) PDF of tilt angle.

$\hat{k}_{r}=\hat{k}_{i}-2\left(\hat{k}_{i} \cdot \hat{z}\right) \hat{z}$ which makes an angle $\beta_{r}=\cos ^{-1}\left(\hat{k}_{r} \cdot \hat{z}^{\prime}\right)$ with the cylinder axis. The reflected field $\left(\overrightarrow{\mathbf{E}}^{r}\right)$ consists of the parallel and perpendicular components of the incident wave, and are modified by the Fresnel reflection coefficients $\left(R_{\|}\right.$and $\left.R_{\perp}\right)$, respectively, i.e., $\overrightarrow{\mathbf{E}}^{r}=E_{v}^{i} R_{\|} \hat{v}_{r}+E_{h}^{i} R_{\perp} \hat{h}_{r}$ where $\hat{v}_{r}$ is a unit vector in the plane of incidence, and perpendicular to $\hat{k}_{r}$ and $\hat{h}_{r}$ is perpendicular to the plane of incidence. The expansion shown in (10), and (11) can be used to find $\overrightarrow{\mathbf{E}}^{g t}$ using $\overrightarrow{\mathbf{E}}^{r}$ as the incident wave on the cylinder. To include other components, approximate image currents in the ground are used. From an asymptotic formulation of the electric, and magnetic Hertz potentials of an infinitesimal dipole above the ground [17], approximate image currents can be inferred as

$$
\begin{aligned}
\overrightarrow{\mathbf{J}}_{e}^{i}= & R_{\perp} a_{x}^{e} \hat{X}+R_{\perp} a_{y}^{e} \hat{Y} \\
& +\left(R_{\|} a_{z}^{e}+\cos \phi R_{1} a_{x}^{e}+\sin \phi R_{1} a_{y}^{e}\right) \hat{Z}
\end{aligned}
$$




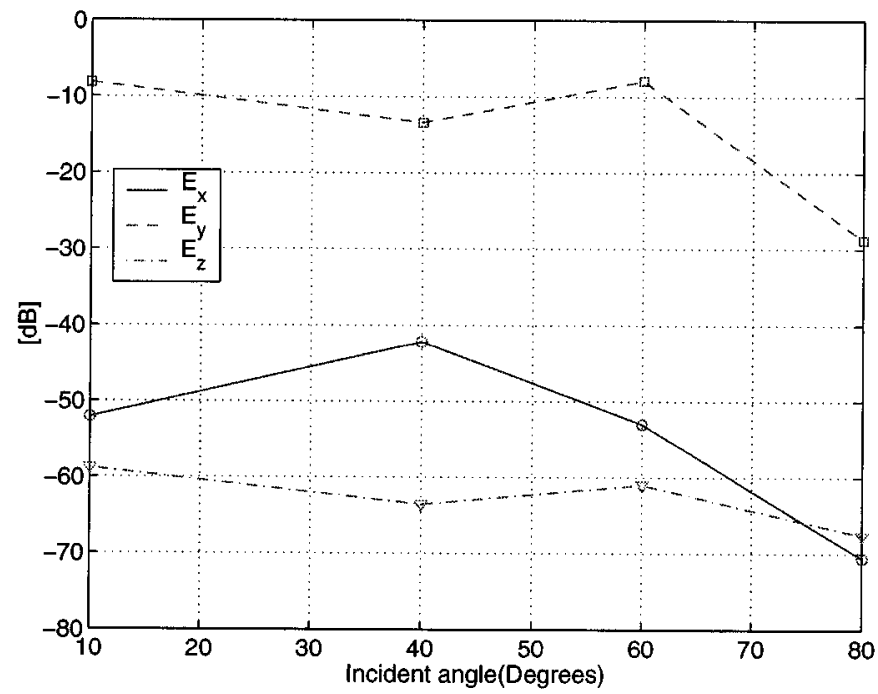

(a)

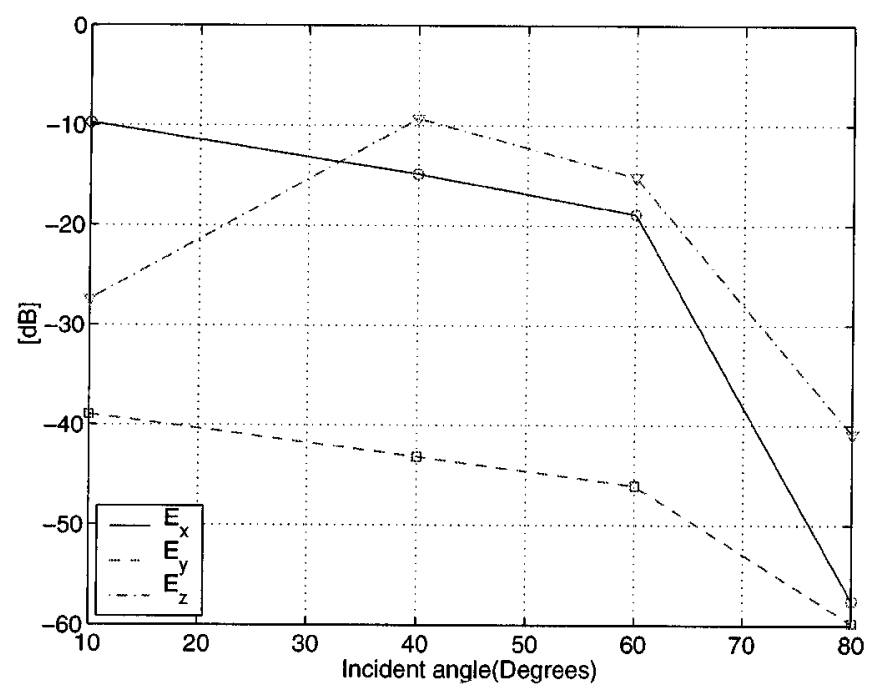

(b)

Fig. 11. Mean of total field at $z=1 \mathrm{~m}$ under deciduous trees as a function of incidence angle. The trees are located randomly with a density, $0.1 / \mathrm{m}^{2}$, and the results include the effects of leaves. (a) The incident wave is $h$-polarized. (b) The incident wave is $v$-polarized.

$$
\begin{aligned}
\overrightarrow{\mathbf{J}}_{m}^{i}= & R_{\|} a_{x}^{m} \hat{X}+R_{\|} a_{y}^{m} \hat{Y} \\
& +\left(R_{\perp} a_{z}^{m}+\cos \phi R_{1} a_{x}^{m}+\sin \phi R_{1} a_{y}^{m}\right) \hat{Z}
\end{aligned}
$$

where the currents above the ground plane are given as $\overrightarrow{\mathbf{J}}_{e}=$ $a_{x}^{e} \hat{X}+a_{y}^{e} \hat{Y}+a_{z}^{e} \hat{Z}$, and $\overrightarrow{\mathbf{J}}_{m}=a_{x}^{m} \hat{X}+a_{y}^{m} \hat{Y}+a_{z}^{m} \hat{Z}$. Here $R_{1}=$ $2 \sin \theta \cos \theta\left(\cos \theta-\sqrt{\varepsilon_{d}-\sin ^{2} \theta}\right) /\left(\varepsilon_{d} \cos \theta+\sqrt{\varepsilon_{d}-\sin ^{2} \theta}\right)$, and $\varepsilon_{d}$ is the dielectric constant of the ground. The reflection coefficients, $R_{\perp}, R_{\|}$, and $R_{1}$ and other quantities such as $\sin \phi$, and $\cos \phi$ can be assumed constant because in the far-field region, the variation of these quantities is very small. The angle at which these Fresnel reflection coefficients are calculated is obtained by using the line connecting the image point to the observation point, that is, if $\vec{r}_{0}$ denotes the observation point and $\vec{r}_{s}^{i}$ represents the image of the source point this angle is defined as $\theta=\cos ^{-1}\left(\hat{z} \cdot\left(\vec{r}_{0}-\vec{r}_{s}^{i}\right) /\left(\left|\vec{r}_{0}-\vec{r}_{s}^{i}\right|\right)\right)$. To compute $\overrightarrow{\mathbf{E}}^{t g}$, and

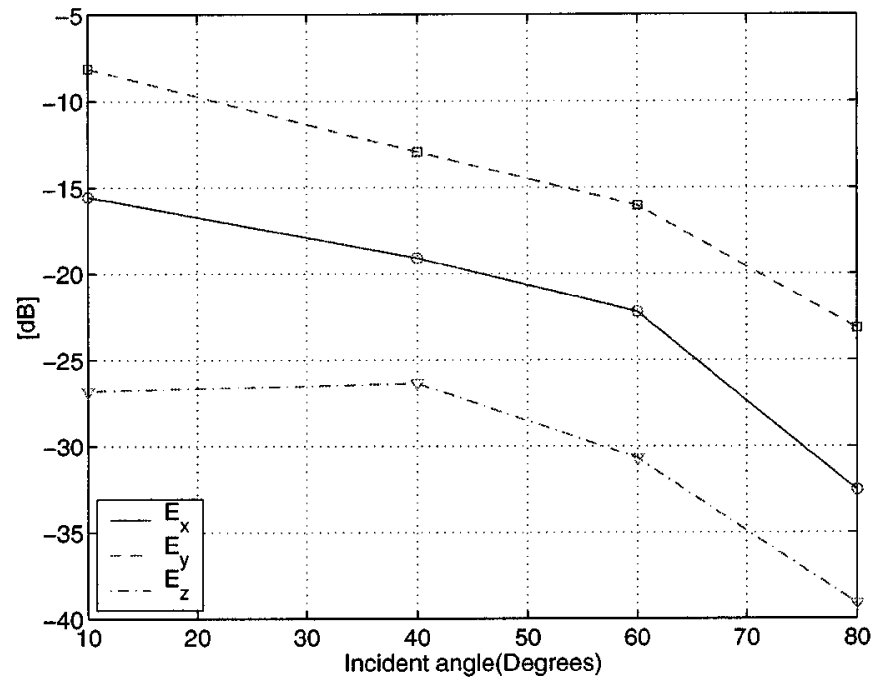

(a)

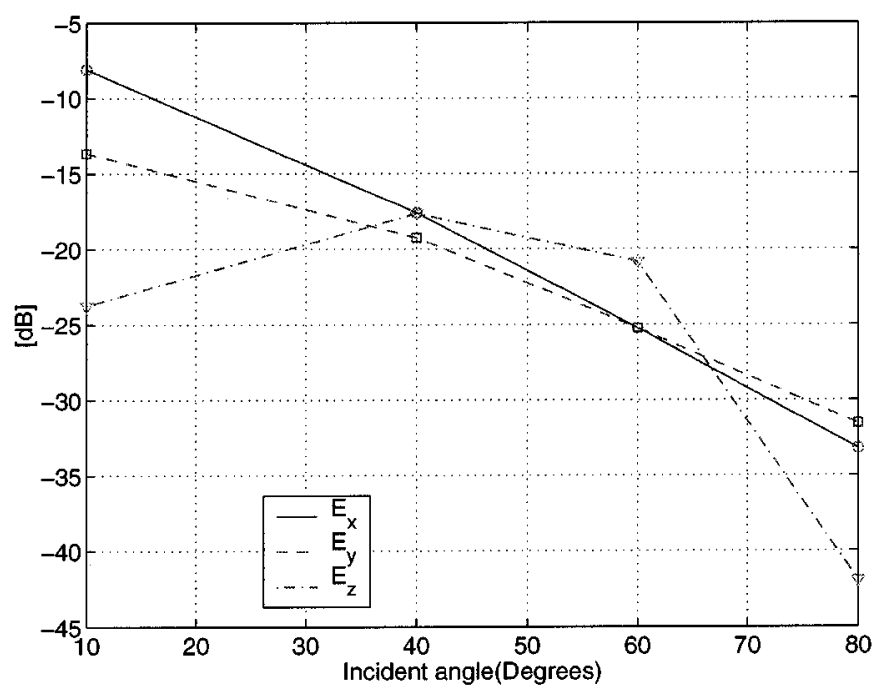

(b)

Fig. 12. Standard deviation of total field at $z=1 \mathrm{~m}$ under deciduous trees as a function of incidence angle. The trees are located randomly with a density, $0.1 / \mathrm{m}^{2}$, and the results include the effects of leaves. (a) The incident wave is $h$-polarized. (b) The incident wave is $v$-polarized.

$\overrightarrow{\mathbf{E}}^{g t g},(10)$, and (11) can now be used but the terms $\vec{C}_{m}$ and $\vec{D}_{m}$ must be modified as

$$
\begin{aligned}
\vec{C}_{m}= & Y_{0} Q_{1 m} R_{\perp}\left(P_{1 m} \hat{x}-P_{2 m} \hat{y}\right)+\left[Q_{1 m} J_{m} R_{\|}\right. \\
& \left.+\left(\cos \phi P_{1 m}-\sin \phi P_{2 m}\right) Y_{0} Q_{1 m} R_{1}\right] \hat{z} \\
\vec{D}_{m}= & \bar{Q}_{1 m} R_{\|}\left(P_{2 m} \hat{y}-P_{1 m} \hat{x}\right)+\left[\bar{Q}_{2 m} J_{m} R_{\perp}\right. \\
& \left.+\left(\sin \phi P_{2 m}-\cos \phi P_{1 m}\right) \bar{Q}_{1 m} R_{1}\right] \hat{z}
\end{aligned}
$$

where $J_{m}=J_{m}\left(y_{0}\right)$.

\section{NUMERICAL SIMULATION}

In this section, the validity of the approximate formulations for the scattered field of a finite cylinder are being examined. A number of approximations were used to derive the integral expression given by (6). Fig. 4 shows the comparison between the 


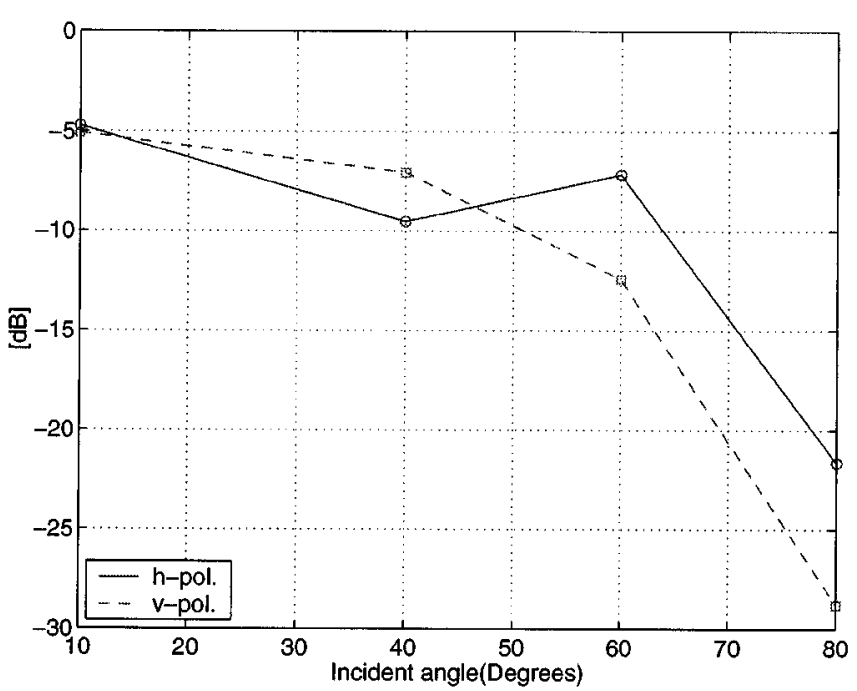

(a)

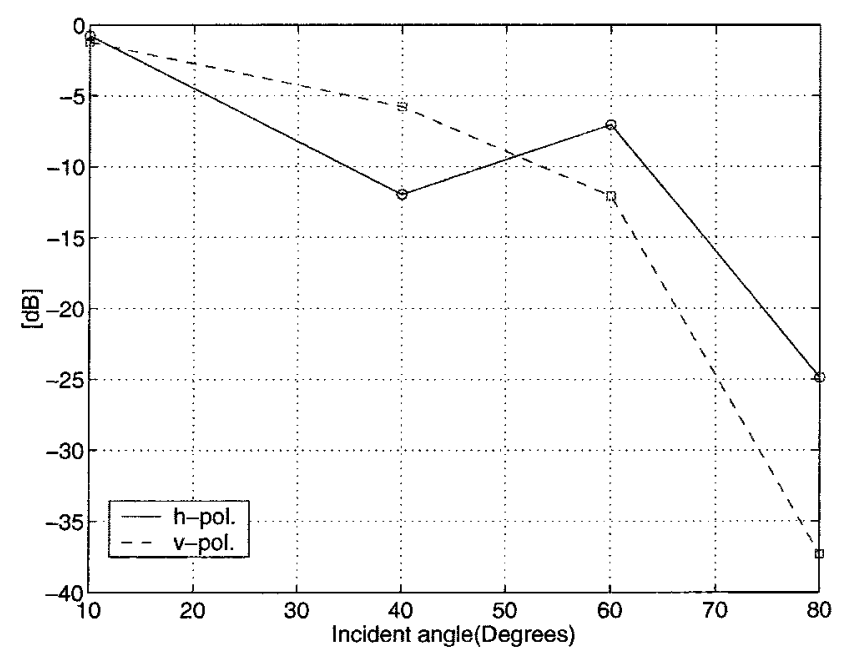

(b)

Fig. 13. Total and received power at $z=1 \mathrm{~m}$ under deciduous trees as a function of incidence angle. The trees are located randomly with a density, $0.1 / \mathrm{m}^{2}$. 'o' and ' $\square$ ' are results that include the effects of leaves. (a) $\left\langle|E|^{2}\right\rangle$ (b) Path loss.

two results from an exact numerical integration and the analytic formulation, (6). In this comparison, $L$ is $4 \lambda$ and the observation point is at $z=L / 3$ and $0.1<\rho<100$. As can be seen the two results are in excellent agreement over the entire region of comparison. The next two (Figs. 5 and 6) show the accuracy of (10) when a TM, and TE waves are incident on a $4 \lambda$ long cylinder with dielectric constant, $5+j$, and a radius, $a=5 \mathrm{~cm}$, at $\beta=60^{\circ}$, and $\phi_{i}=180^{\circ}$. For the TM wave incidence case, only one principal component $\left(E_{x}\right)$ is plotted for brevity, but the same accuracy is observed for the other component $\left(E_{z}\right)$. A MoM solution [11] is used for exact calculation of the scattered field. It is shown that the two results are in good agreement. The accuracy of the approximate formulation (10) degrades as the ratio (radius/length) is increased.

The developed forest channel model is used to demonstrate performance assessment of GPS receivers placed under coniferous and deciduous canopies. For these simulations the

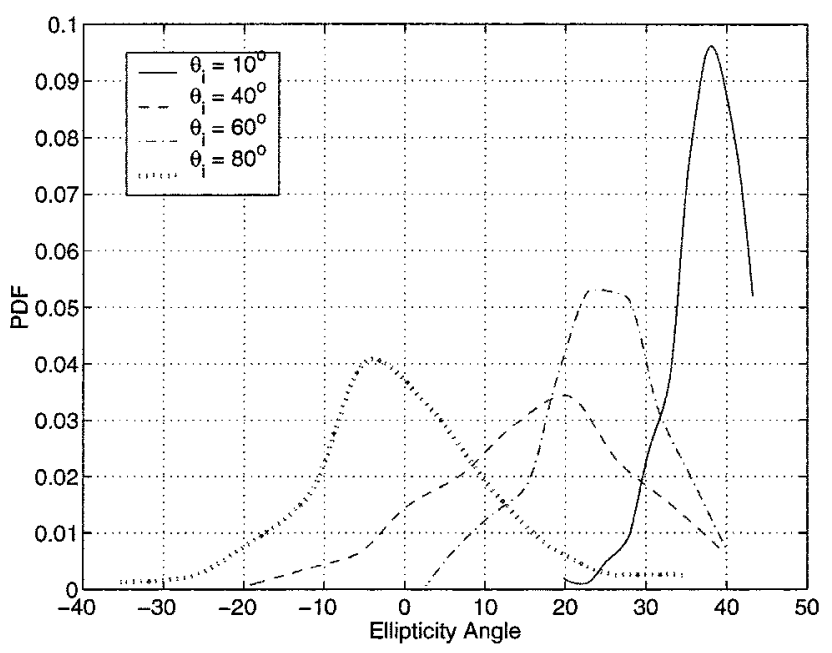

(a)

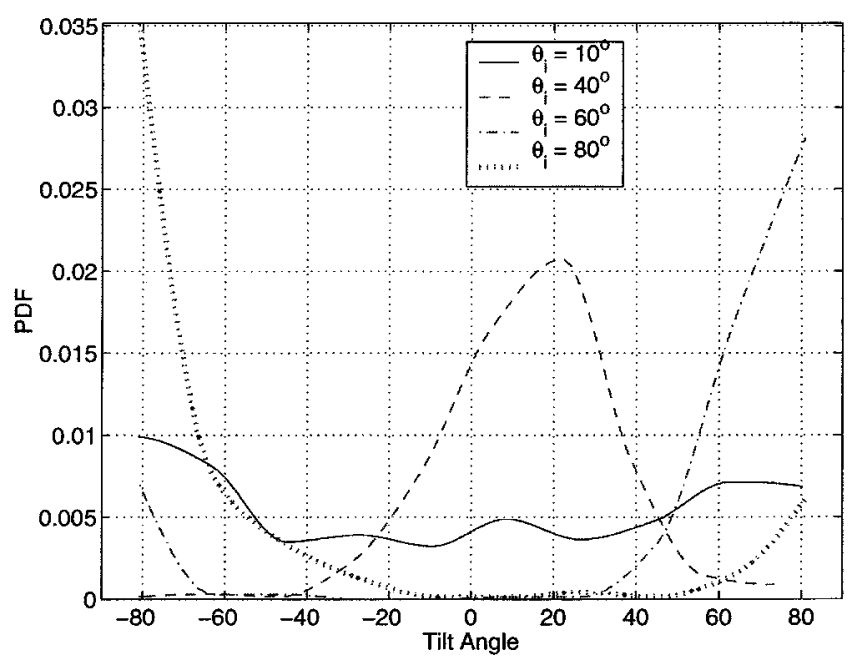

(b)

Fig. 14. pdf of polarization of the received signal at $z=1 \mathrm{~m}$ under deciduous trees. Four results are plotted whose incidence angles are $10^{\circ}, 40^{\circ}, 60^{\circ}$, and $80^{\circ}$. (a) pdf of ellipticity angle. (b) pdf of tilt angle.

frequency is set at $1.57542 \mathrm{GHz}$ and the polarization is chosen to be right-hand circular (RHCP). For the following simulations a receiver is placed $1 \mathrm{~m}$ above a ground plane having a soil moisture content $m_{v}=0.18$ which consists of $50 \%$ sand and $10 \%$ clay. At this GPS frequency the soil dielectric constant is calculated to be $\varepsilon_{s}=9.75+j 1.62$ [6]. To derive the field statistics and forest channel properties, a Monte Carlo simulation is carried out using 400 realizations. To account for near-field effects, 10 trees randomly located around the receiver are included in the simulations. First, a relatively young red pine stand with average tree height of $9.5 \mathrm{~m}$ and the density of $0.1 \mathrm{trees} / \mathrm{m}^{2}$ is considered. The mean and standard deviation of the total field (incident + reflected + scattered) are shown in Figs. 7, and 8, respectively, for both $h$ - and $v$-polarized incident waves as function of incidence angle. As the incidence angle increases, the field is more attenuated as expected and small cross-polarized components are observed. In Fig. 7(a), the mean-field jumps approximately $10 \mathrm{~dB}$ 


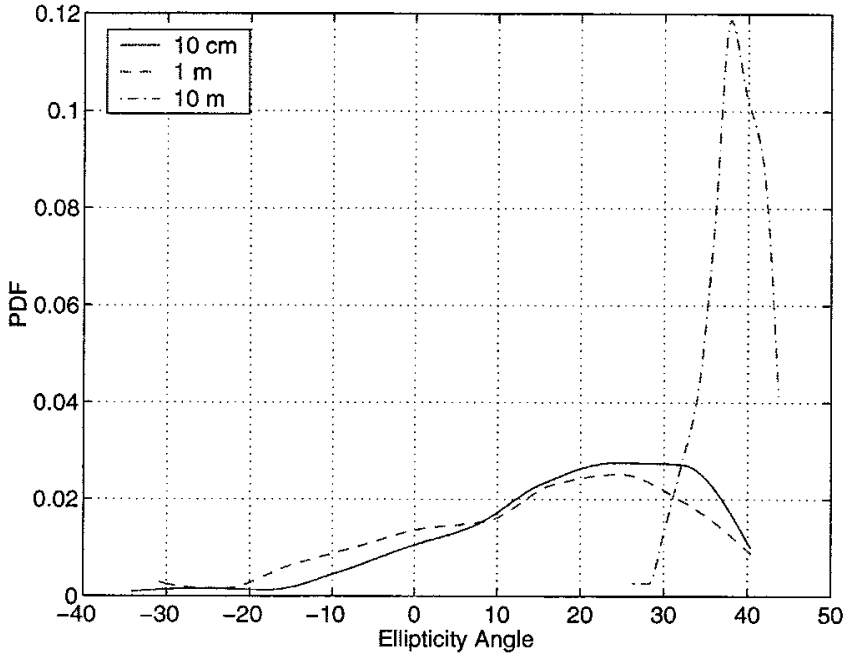

(a)

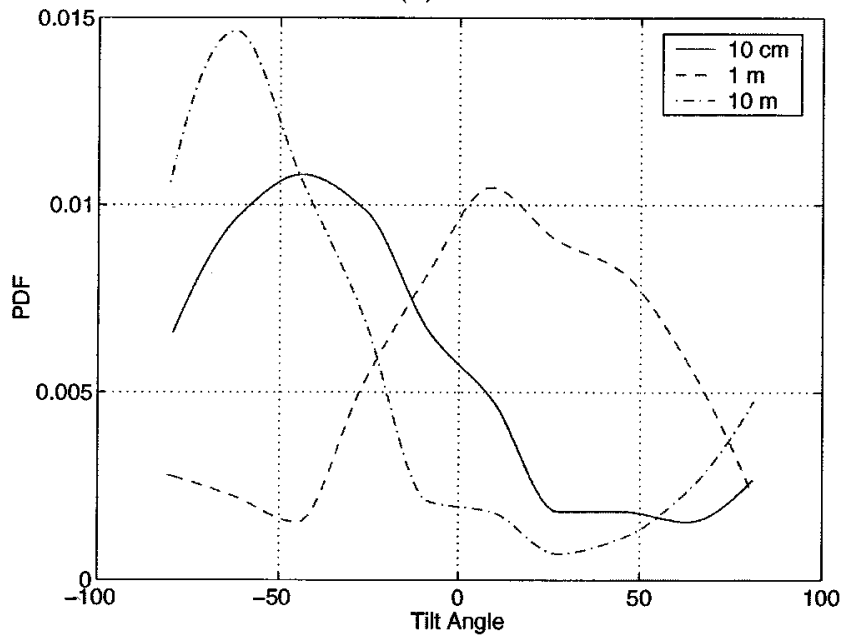

(b)

Fig. 15. pdf of polarization of the received signal at $z=1 \mathrm{~m}$ under pine trees. Three results are plotted whose receiver heights are $0.1,1$, and $10 \mathrm{~m}$. (a) PDFof ellipticity angle. (b) PDF of tilt angle.

TABLE I

Mean of Total Field Under Pine Trees at Three Different Heights, $0.1 \mathrm{~m}, 1 \mathrm{~m}$, AND $10 \mathrm{~m}$ When a Plane WaVe Is INCIDENT AT $40^{\circ}$

\begin{tabular}{c|c|c|c|c|c|c}
\hline & \multicolumn{3}{|c|}{ h-pol. incident } & \multicolumn{3}{c}{ v-pol. incident } \\
\hline Height & $E_{x}[\mathrm{~dB}]$ & $E_{y}[\mathrm{~dB}]$ & $E_{z}[\mathrm{~dB}]$ & $E_{x}[\mathrm{~dB}]$ & $E_{y}[\mathrm{~dB}]$ & $E_{z}[\mathrm{~dB}]$ \\
\hline $10 \mathrm{~cm}$ & -39.9 & -5.4 & -45.5 & -7.4 & -33.6 & -6.2 \\
\hline $1 \mathrm{~m}$ & -46.5 & -10.1 & -41.3 & -10.1 & -39.7 & -5.1 \\
\hline $10 \mathrm{~m}$ & -44.9 & 0.8 & -49.9 & -1.6 & -45.7 & -4.3 \\
\hline
\end{tabular}

at incidence angle $50^{\circ}$ due to the interference between the direct incident wave and the reflected wave from the ground. Note that $E_{z}$ has more fluctuation that $E_{x}$ and $E_{y}$. This is due to the fact that most scattering fluctuations are caused by the tree trunks which are big and vertically alligned. Fig. 9 shows the path loss as a function of incidence angle. The path loss is defined as the ratio of the total power received to that in the free space. In this example, a short dipole aligned to receive the incident wave is
TABLE II

Standard Deviation of Total Field Under Pine Trees at Three DifFERENT HeIGHTS, $0.1 \mathrm{~m}, 1 \mathrm{~m}$, AND $10 \mathrm{~m}$ When a Plane WAVE IS INCIDENT AT $40^{\circ}$

\begin{tabular}{c|c|c|c|c|c|c}
\hline & \multicolumn{3}{|c|}{ h-pol. incident } & \multicolumn{3}{c}{ v-pol. incident } \\
\hline Height & $E_{x}[\mathrm{~dB}]$ & $E_{y}[\mathrm{~dB}]$ & $E_{z}[\mathrm{~dB}]$ & $E_{x}[\mathrm{~dB}]$ & $E_{y}[\mathrm{~dB}]$ & $E_{z}[\mathrm{~dB}]$ \\
\hline $10 \mathrm{~cm}$ & -14.2 & -6.9 & -15.7 & -8.6 & -14.4 & -12.6 \\
\hline $1 \mathrm{~m}$ & -13.2 & -6.4 & -14.7 & -7.8 & -13.2 & -9.9 \\
\hline $10 \mathrm{~m}$ & -21.7 & -15.6 & -23.0 & -17.2 & -21.4 & -22.6 \\
\hline
\end{tabular}

TABLE III

Total and Received Power Under Pine TReEs at Three DifFerent HeIGHTS, $0.1 \mathrm{~m}, 1 \mathrm{~m}$, AND $10 \mathrm{~m}$ When a Plane WaVe Is INCIDENT at $40^{\circ}$.

\begin{tabular}{c|c|c|c|c}
\hline & \multicolumn{2}{|c|}{ Total power } & \multicolumn{2}{c}{ Received power } \\
\hline Height & h-pol.[dB] & v-pol.[dB] & h-pol.[dB] & v-pol.[dB] \\
\hline $10 \mathrm{~cm}$ & -2.6 & -1.8 & -3.1 & -2.8 \\
\hline $1 \mathrm{~m}$ & -3.9 & -1.4 & -4.8 & -2.4 \\
\hline $10 \mathrm{~m}$ & 0.9 & 0.4 & 0.9 & 0.2 \\
\hline
\end{tabular}

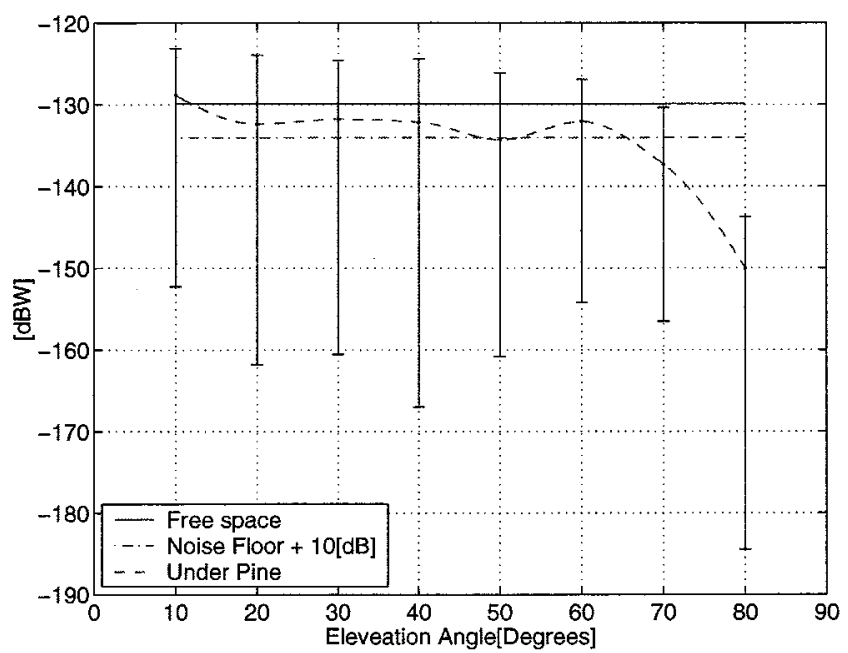

(a)

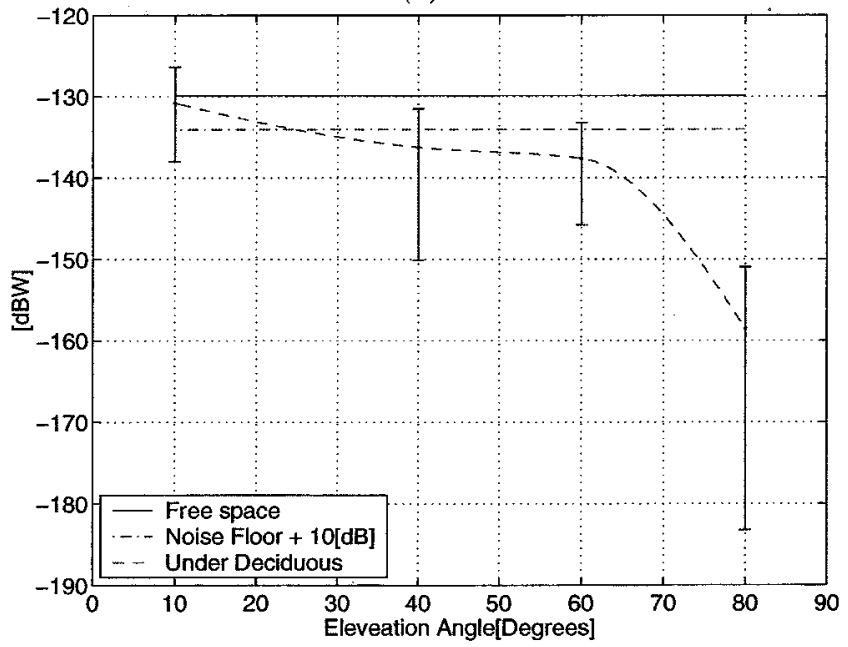

(b)

Fig. 16. Plot of the received signal power under pine and deciduous forest with a density of $0.1 / \mathrm{m}^{2}$ and the received signal in free space. ' $\mathrm{I}$ ' is the range of the received power. (a) Under pine trees. (b) Under deciduous trees. 
used as the receiver antenna. Next, we examine the statistics of the polarization of the received signal. Polarization of a wave is characterized by two parameters, the ellipticity angle $\chi$ and the tilt angle, $\xi[18]$. At the receiver both the vertical and horizontal components of the electric field in a plane perpendicular to the direction of incidence are calculated and for which $\chi$ and $\xi$ are evaluated. In this simulation the polarization of incidence wave is assumed be right hand circular polarization. A Monte Carlo simulation is used to estimate the pdf of $\chi$ and $\xi$ for the red pine stand. It is shown that as the incidence angle is increased the polarization of the received signal is modified, however, it remains always righthanded. A similar Monte Carlo simulation is carried out for deciduous forest stand that consist of red maples. A tree density of $0.1 / \mathrm{m}^{2}$ and average height of $17 \mathrm{~m}$ and trunk diameter of $20 \mathrm{~cm}$ is chosen. This stand has a dry biomass of $107 \mathrm{~kg} /$ tree. Simulations for both foliated and defoliated trees are performed. Figs. 11, and 12 show the mean field and standard deviation for a receiver $1 \mathrm{~m}$ above the ground for a foliated stand as a function of incidence angle. The same jump phenomenon is observed in the mean field due to the same interference as the pine tree case. Similar to the pine forest simulation, the standard deviation is comparable to or larger than the mean field, and the level of the cross polarized filed is relatively low. The path loss is also calculated and shown in Fig. 13. As the incidence angle increases, the $v$-polarized wave is more attenuated because the path length increases in the trunk layer where scatterers are aligned mostly along $\hat{z}$-axis. Whereas the number of leaves (of the order of 12000 per a typical tree) is higher than the number of branches and twigs $(\sim 7000)$, scattering from leaves can be ignored as their typical dimension $(7 \mathrm{~cm})$ is small compared to the wavelength. However, leaves can cause considerable attenuation. Fig. 14 shows the pdf of ellipticity angle and tilt angle for a receiver $1 \mathrm{~m}$ above ground under the red maple stand. It is shown that the incident wave approached a linear wave ( $h$-polarized).

Next, we examine the effect of receiver height on the field attenuation, fluctuation, and depolarization for the red pine stand at $40^{\circ}$ incidence. Tables I and II show the three components of mean field and its standard deviation for the two principal polarizations. It is shown that the ratio of standard deviation to mean field decreases as the receiver height is increased. Table III shows the copolarized path loss as a function of receiver height. The pdf of polarization state as a function of receiver height for incidence angle $40^{\circ}$ are shown in Fig. 15. The polarization at $z=10 \mathrm{~m}$ is almost the same as that of the incident wave (RHCP).

Now let us consider a GPS receiver at a height of $1 \mathrm{~m}$ above ground embedded in the red pine. It is assumed that polarization of the antenna is RHCP and has a free space gain of $0 \mathrm{dBi}$. The power received by such receiver in free space is $-160 \mathrm{dBW}$ on the earth surface [2]. This signal level is below the thermal noise power at the receiver if it were not for the processing gain of about $30 \mathrm{~dB}$ for the 1023 chip code length of the GPS receiver [19]. Considering the receiver bandwidth is $B=2.046 \mathrm{MHz}$, the noise power is calculated from

$$
N=K T B=K T_{0}(N F-1) B \approx-134 \mathrm{dBW} .
$$

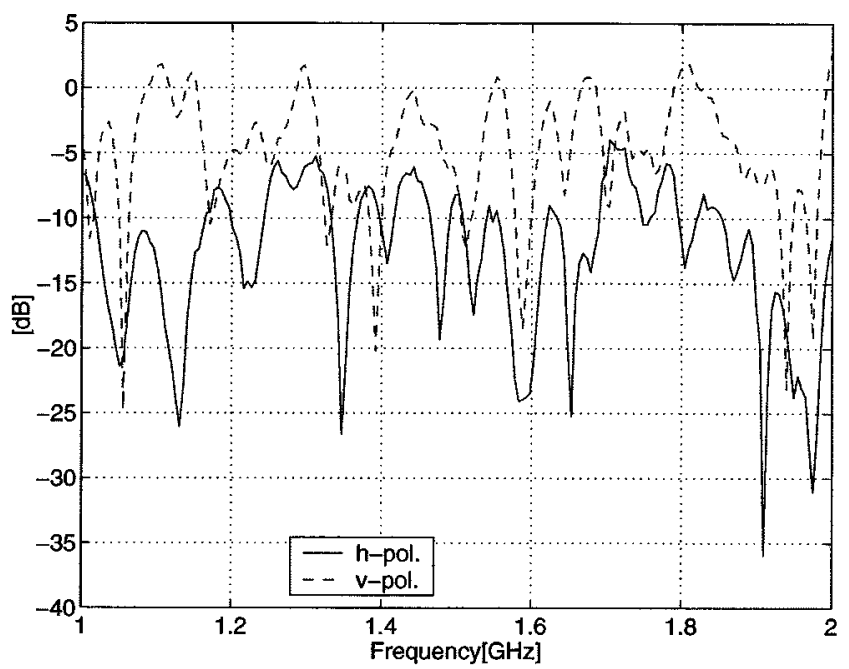

(a)

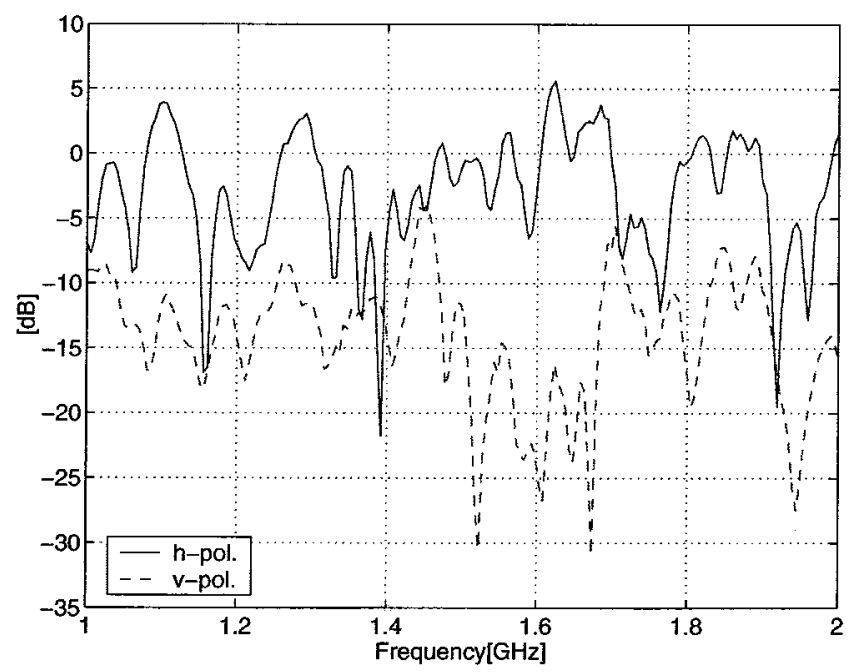

(b)

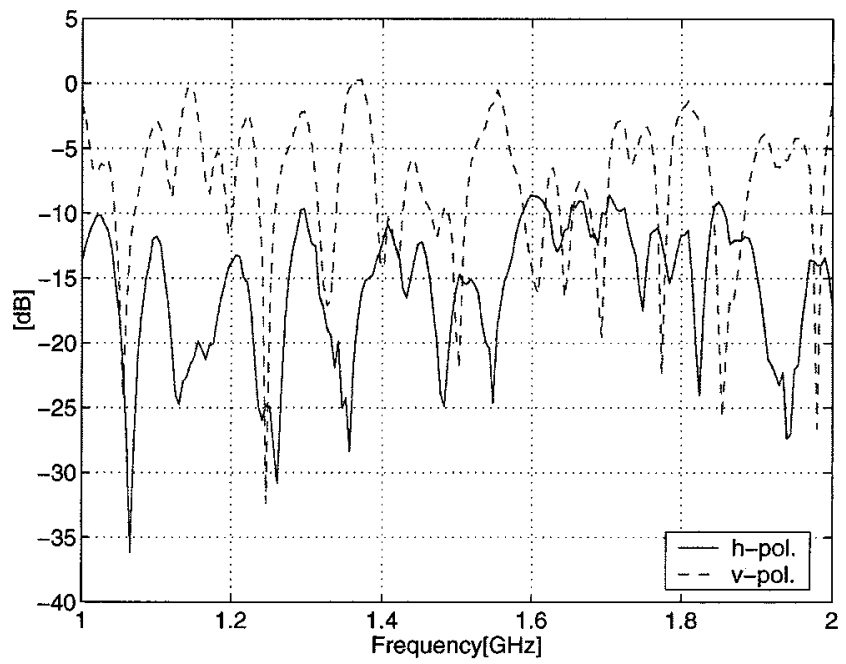

(c)

Fig. 17. Frequency response of a pine forest over $1-\mathrm{GHz}$ bandwidth (1-2 $\mathrm{GHz}$ ) when a plane wave is incident $40^{\circ}$. The receiver is $1 \mathrm{~m}$ over the ground plane. Effect of 20 trees near a receiver. (a) $E_{x}$. (b) $E_{y}$. (c) $E_{z}$. 


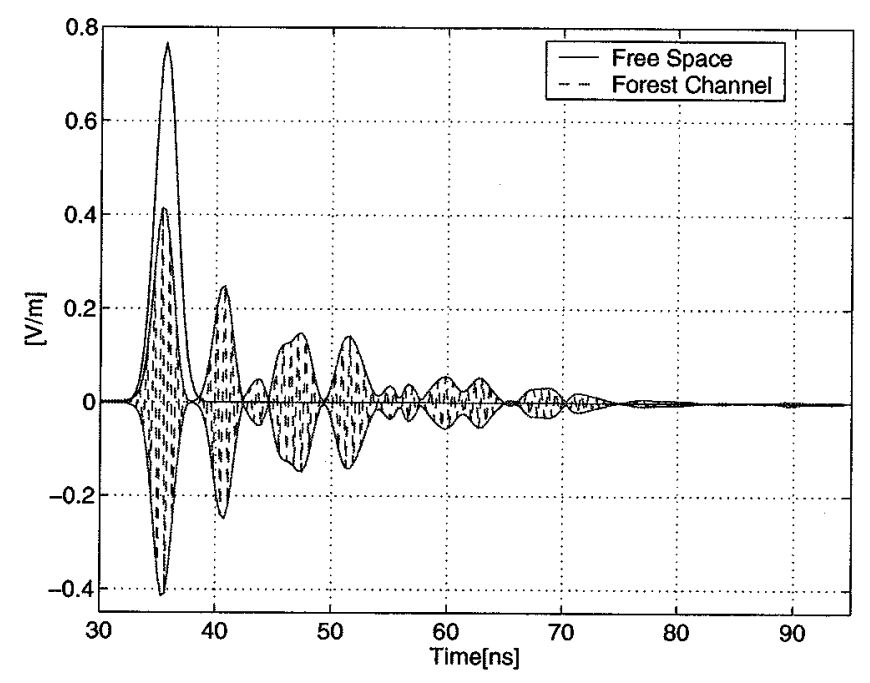

(a)

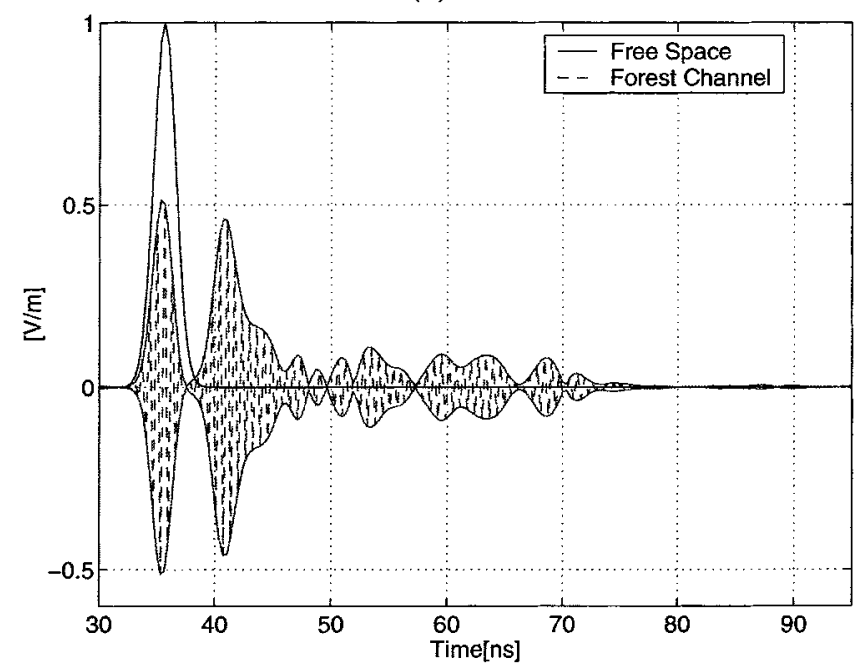

(b)

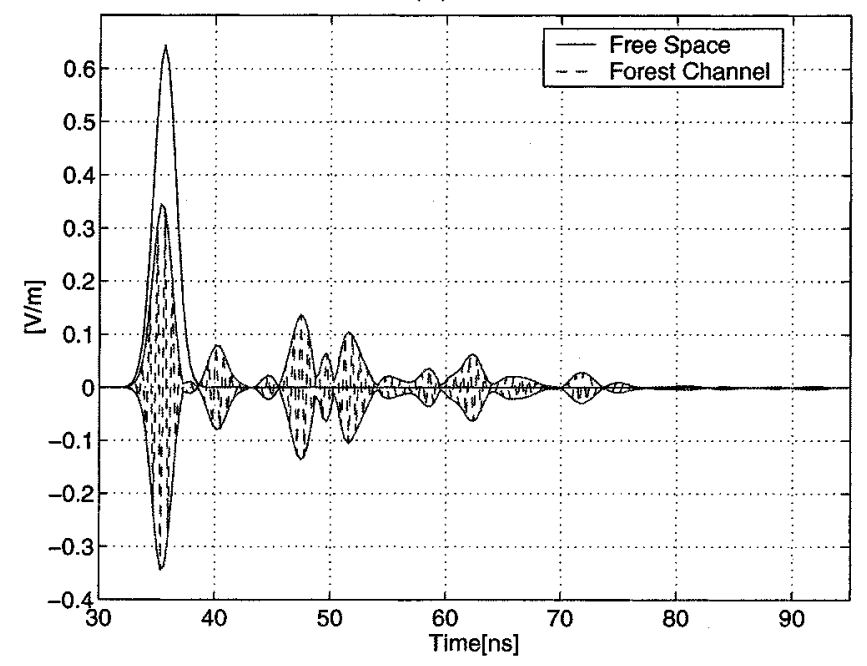

(c)

Fig. 18. Time-domain response of a narrow pulse transmission through a pine forest. The pulse is incident at $40^{\circ}$. (a) $E_{x}$. (b) $E_{y}$. (c) $E_{z}$.

This calculation assumes a receiver noise figure of $1.5 \mathrm{~dB}$ and the noise power associated with the antenna temperature is ignored. However, it should be pointed out that the antenna tem- perature operating under foliage can be significantly different (higher) than that without foliage. Fig. 16(a) shows the mean power received by the GPS receiver under red pine forest. Also shown in this figure (the vertical bar) is a power range of the simulation and the received power in the absence of foliage, and the thermal noise power plus $10 \mathrm{~dB}$ (threshold $S / N$ ). It is shown that the mean signal power is above the threshold for incidence angles below $70^{\circ}$, however, strong power fluctuations may hamper receiver signal lock on a given satellite. Fig. 16(b) shows the performance of the same receiver below the red maple stand. Here receiver power fluctuation is less since the lower branches are farther from the receivers. In both cases reliable, operation cannot be guaranteed. To remedy this problem more transmitter power (by a factor of $15 \mathrm{~dB}$ ) is needed.

Last we examine the performance of a wide-band time domain communication system. Time-domain modulation using very narrow pulses is being considered for military applications, since it minimizes evesdropping and provides antijamming capability. A signal occupying a bandwidth $1-2 \mathrm{GHz}$ is considered. Again a receiver is considered to be $1 \mathrm{~m}$ above the ground plane in the red pine stand. An incidence angle of $40^{\circ}$ is assumed. Fig. 17 shows the frequency response of three components (for a given realization of tree) for both vertical and horizontal incident wave. Strong spectral field fluctuations are a result of multipath caused by strong scatterers in the channel. The Fourier transform of these responses provides the time domain response of the channel. Fig. 18 shows the time domain response for the three received field components assuming the incident field has a Gaussian envelope for both the vertical and horizontal incident waves.

\section{CONCLUSION}

A physics-based channel estimation model for a forested environment is developed. This model is appropriate when either the transmitter or receiver is within the canopy and the other is outside. Realistic tree structures are constructed using a fractal algorithm and scattering from individual tree components are calculated and added coherently. An accurate approximate uniform near-field to far-field scattering solution for dielectric cylinders representing branches and tree trunks is also developed and incorporated into the tree model. Monte Carlo simulations are carried out in order to estimate the statistics of the channel behavior, such as path loss, coherence bandwidth, field fluctuation, etc. In specifics, performance of GPS receivers under foliage is evaluated and it is shown that the signal attenuation, depolarization, and field fluctuation hamper proper operation of a receiver under both coniferous and deciduous stands with heights as low as $10 \mathrm{~m}$. The same model is used to assess the performance of wide-band communications.

\section{REFERENCES}

[1] P. Enge and P. Misra, "Special issue on global positioning system," Proc. IEEE, vol. 87, pp. 3-15, Jan. 1999.

[2] Global Positioning System Standard Positioning Service Signal Specification, 1995.

[3] H. L. Bertoni, Radio Propagation for Modern Wireless Systems. Upper Saddle River, NJ: Prentice-Hall, 2000.

[4] K. Sarabandi, "Electromagnetic Scattering from Vegetation Canopies," Ph.D dissertation, University of Michigan, Ann Arbor, 1989. 
[5] K. Sarabandi, P. F. Polatin, and F. T. Ulaby, "Monte Carlo simulation of scattering from a layer of vertical cylinder," IEEE Trans. Antennas Propagat., vol. 41, pp. 465-475, Apr. 1993.

[6] Y. C. Lin and K. Sarabandi, "Monte Carlo coherent scattering model for forest canopies using fractal-generated trees," IEEE Trans. Geosci. Remote Sensing, vol. 37, pp. 440-451, 1999.

[7] M. A. Karam, A. K. Fung, and Y. M. M. Antar, "Electromagnetic wave scattering from some vegetation samples," IEEE Trans. Geosci. Remote Sensing, vol. 26, pp. 799-808, 1988.

[8] P. L. E. Uslenghi, "The backscattering radar cross section of long thin dielectric bodies of revolution on a metal plane," Can. J. Phys., vol. 43, pp. 1164-1167, 1965.

[9] T. Tamir, "On radio-wave propagation in forest environments," IEEE Trans. Antennas Propagat., vol. AP-15, pp. 806-817, Nov. 1967.

[10] K. Sarabandi and I. Koh, "Effect of canopy-air interface roughness on HF-UHF wave propagation in forest," IEEE Trans. Antennas Propagat., vol. 50, pp. 111-121, Feb. 2002.

[11] _ - "A complete physics-based channel parameter simulation for wave propagation in a forest environment," IEEE Trans. Antennas Propagat., vol. 49, pp. 260-271, Feb. 2001.

[12] L. L. Foldy, "The multiple scattering of waves," Phys. Rev., vol. 67, pp. $107-119,1945$

[13] L. B. Felsen and N. Marcuvitz, Radiation and Scattering of Waves. New York: Prentice-Hall, 1973.

[14] R. E. Collin, Field Theory of Guided Waves. New York: IEEE Press, 1990.

[15] Handbook of Mathematical Functions, Dover, New York, 1965

[16] R. Schiffer and K. O. Thielheim, "Light scattering by dielectric needles and disks," J. Appl. Phys., vol. 50, no. 4, pp. 2476-2483, Apr. 1979.

[17] R. E. Collin and F. J. Zucker, Antenna Theory. New York: McGrawHill, 1969.

[18] F. T. Ulaby, R. K. Moore, and A. K. Fung, Microwave Remote Sensing Active and Passive. Norwood, MA: Arthech House, 1982.

[19] J. G. Proakis, Digital Communications. New York: McGraw-Hill, 1989.

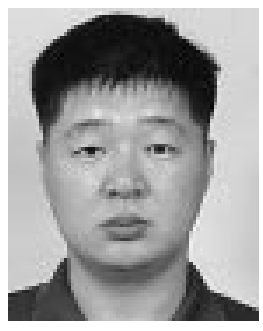

Il-Suek Koh (S'01-A'02) was born in Korea. He received the B.S. and M.S. degrees in electronics engineering from Yonsei University, Seoul, Korea, in 1992 and 1994, respectively. He is currently working toward the Ph.D. degree at the University of Michigan, Ann Arbor.

In 1994, he joined LG Electronics Ltd. as an Assistant Research Engineer. His research interests are theoretical modeling of wireless communication channel, multibody problems, and numerical techniques for electrically large objects.

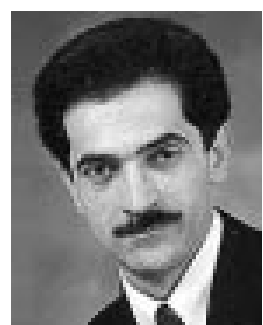

Kamal Sarabandi (S'87-M'90-SM'9-F'00) received the B.S. degree in electrical engineering from Sharif University of Technology, Tehran, Iran, in 1980. He received the M.S.E. degree in electrical engineering, the M.S. degree in mathematics, and the $\mathrm{Ph} . \mathrm{D}$. degree in electrical engineering, from the University of Michigan, Ann Arbor, in 1986, 1989, and 1989 , respectively.

$\mathrm{He}$ is the Director of the Radiation Laboratory and Professor in the Department of Electrical Engineering and Computer Science, University of Michigan. His research areas of interest include electromagnetic wave propagation, antennas, and microwave and millimeter-wave radar remote sensing. He has served as the Principal Investigator on many projects sponsored by NASA, JPL, ARO, ONR, ARL, NSF, DARPA, and numerous industries. He has published many book chapters and more than 90 papers in refereed journals on electromagnetic scattering, random media modeling, wave propagation, antennas, microwave measurement techniques, radar calibration, inverse scattering problems, and microwave sensors. He has more than 170 papers and invited presentations in national and international conferences and symposia on similar subjects.

Dr. Sarabandi is a member of the IEEE Geoscience and Remote Sensing Society (GRSS) ADCOM, Chairman of the Awards Committee of the IEEE GRSS, and a member of IEEE Technical Activities Board Awards Committee. He is serving as the Associate Editor of the IEEE TRANSACTIONS ON ANTENNAS AND PROPAGATION and the IEEE SENSORS JOURNAL. He is also a member of Commission F of URSI and of the Electromagnetic Academy. He is listed in American Men \& Women of Science, Who's Who in America, and Who's Who in Electromagnetics. He was the recipient of the prestigious Henry Russel Award from the Regent of the University of Michigan (the highest honor the University of Michigan bestows on a faculty member at the assistant or associate level). In 1999, he received a GAAC Distinguished Lecturer Award from the German Federal Ministry for Education, Science, and Technology. He was also a recipient of a 1996 Teaching Excellence Award from the EECS Department of the University of Michigan. In the past several years, joint papers presented by his students at a number of symposia (IEEE AP'95, '97, '00, '01, IEEE MTT-S'01, and IEEE IGARSS'99) have received student prize paper awards. 